\title{
Numerical simulation of vortex instabilities in the wake of a pre-swirl pumpjet propulsor
}

\author{
Denghui Qin, ${ }^{1,2}$ Qiaogao Huang, $, 1,2$, a) Guang Pan, ${ }^{1,2}$ Peng Han, ${ }^{3}$ Yang Luo, ${ }^{4}$ and Xinguo Dong ${ }^{5}$ \\ ${ }^{1)}$ School of Marine Science and Technology, Northwestern Polytechnical University, Xi'an 710072, \\ China \\ ${ }^{2)}$ Key Laboratory for Unmanned Underwater Vehicle, Northwestern Polytechnical University, Xi'an 710072, \\ China \\ ${ }^{3)}$ Department of Mechanics, LadHyX, CNRS, École Polytechnique, 91128 Palaiseau, France \\ 4) Department of Naval Architecture, Ocean and Marine Engineering, University of Strathclyde, Glasgow G4 OLZ, \\ United Kingdom \\ ${ }^{5}$ Science and Technology on Water Jet Propulsion Laboratory, Marine Design and Research Institute of China, Shanghai 200011, \\ China
}

A numerical analysis based on detached eddy simulations is conducted to investigate vortex instabilities in the wake of a pre-swirl pumpjet propulsor. Three models are established to separate the roles that the rotor, stator, and duct play in the vortex structure of the pumpjet propulsor. In this paper, only the vortex structure of the rotor is considered. The results show that the vortex system of the rotor is mainly composed of the tip vortices, a hub vortex, the trailing tip vortices, and the trailing root vortices. The trailing tip vortices are generated by the premature shedding of the tip vortices in the rotor model compared with a normal single propeller. The existence of trailing root vortices increases the stability of the hub vortex. Furthermore, a unique multi-inductance instability mode of the tip vortex, called the "overlap-forward" phenomenon, is found for low values of the advance coefficient $J$. It is found that the instability of the tip vortex depends not only on the spiral-to-spiral distance but also on the highest-efficiency point of the propeller. The instability inception point of the tip vortex moves farther downstream with increasing $J$, whereas when $J$ is greater than the highest-efficiency point of the propeller, the stable length of the tip vortices drops sharply. The energy transfer process from blade harmonics to shaft harmonics of the tip vortices depends on $J$ and is related to the spatial evolution of the tip vortices.

\section{INTRODUCTION}

In recent years, more and more attention has been paid to research on propeller wake flow. Understanding the wake flow and wake instability mechanisms associated with marine propellers is important for naval engineering applications owing to their direct relationship with propulsion, vibration, and noise performance of these propellers.?

There have been many studies of propeller wake dynamics, both theoretical and experimental. In terms of theoretical research, Joukowsky ${ }^{\text {" }}$ was the first to propose a vortex model for a two-bladed propeller. Since then, much theoretical work has been done on wake instability mechanisms.? ? ? ? ? ? ? ? ? As summarized by Kumar and Mahesh, ${ }^{?}$ the vortex system of a typical $N$-bladed rotor is composed of $N$ helical tip vortices, one hub vortex, and $N$ trailing edge vortices. The trailing edge vortices, shedding from the trailing edge of each blade, connect the tip vortex and hub vortex.

There have also been a number of important experimental studies of propeller wake dynamics. Felli and co-workers have done a lot of work in this area. They conducted hydrodynamic experiments on an INSEAN (Italian National Ship Model Basin) E779A model propeller in the Italian Navy Cavitation Tunnel. The flow in the wake of the E779A was measured by both particle image velocimetry (PIV) ${ }^{?}$ and laser Doppler velocimetry (LDV). ${ }^{?}$ In addition, they performed visualizations

\footnotetext{
a) Author to whom correspondence should be addressed: huangqiaogao@nwpu.edu.cn.
}

of wake vortex structures using high-speed cameras. ' They also carried out some water tunnel experiments on other propellers, such as the seven-bladed E1619 model ${ }^{?}$ and a fourbladed ducted propeller? ${ }^{?}$ using LDV and PIV. The propeller wake dynamics were investigated in all of these experiments.

Recent improvements in computer performance have allowed detailed numerical simulations of wake vortices of marine propellers. Computational fluid dynamics (CFD) model is a good candidate to capture information in the flow field, and are increasingly being used to study the wake dynamics of marine propellers. Large eddy simulation (LES) has been applied to simulation of propeller vortex dynamics. $\mathrm{Ku}-$ mar and Mahesh? performed wall-resolved LES for a fivebladed marine propeller (DTMB 4381). Posa et al. ${ }^{\text {' }}$ carried out wall-resolved LES simulations using wall-adapting local eddy viscosity (WALE) subgrid model of a seven-bladed notional propeller (INSEAN E1658). The main structures of tip vortices and hub vortices were revealed with high precision. Of course, LES is expensive in terms of computing resources, owing to the relatively large total number of grids ( 840 million nodes) required. The calculations are also time-consuming. Consequently, detached eddy simulation (DES) is now being adopted as a feasible approach for studying wake dynamics, with the flow near walls being calculated by Reynoldsaveraged Navier-Stokes (RANS) turbulence modeling. Muscari et al. ${ }^{?}$ compared RANS and DES for the simulation of the wake flow of the E779A propeller. Furthermore, DES was used by Di Mascio et al. ${ }^{\text {? }}$ to study the wake dynamics of the E779A propeller operating in drift. In addition, Hu et al. studied the vortex-rudder interactions behind the E779A propeller using both DES and LES. It was shown that the DES ap- 
proach has the ability to track the onset of vortex instabilities and its results are in excellent agreement with experimental observations.

In addition to single propellers, research on wake dynamics has also been extended to new types of propellers, such as ducted propellers. Felli et al. ${ }^{\text {? }}$ carried out an experiment with a ducted propeller in a cavitation tunnel, in which they investigated the impingement of the swirling jet generated by the propeller against a wall. The jet-wall interaction was examined in detail using PIV and a high-speed camera. Gong et $a l .{ }^{?}$ compared wake vortex evolution for a ducted and a nonducted propeller using DES. Compared with non-ducted propellers, the duct was found to have a significant effect on the morphology of the propeller wake vortices.

In this paper, the principal object of study is the pumpjet propulsor (PJP). This is a new type of underwater propulsor that is coming into widespread use for underwater vehicles owing to its excellent hydrodynamic and noise performance. Although a considerable amount of experimental research has been done on the hydrodynamic performance of PJPs? ? ? and by CFD,? ? ? ? there have been relatively few studies of the wake dynamics of PJPs. Specifically, Li et al. ${ }^{?}$ compared different hybrid RANS/LES approaches to the simulation of the wake flow of a pre-swirl PJP. They discussed the effect of the duct on tip vortices, but did not consider the effect of pre-swirl stators or the mechanisms of instability associated with PJPs in any great depth. Qin et al. ${ }^{?}$ applied DES to a post-swirl PJP and compared the vortex structures resulting from a normal PJP and from a PJP with a sawtooth duct. In discussing their results, they focused mainly on the effect of the sawtooth structure on the wake vortices from the PJP.

Up to now, most research on the wake dynamics of marine propellers has focused on single propellers and ducted propellers, and less attention has been paid to the study of PJPs. In fact, the vortex instability mechanism associated with PJPs has yet to be described in the literature.

In the work described in the present paper, a numerical study is carried out to investigate the vortex instabilities in the wake of a pre-swirl pumpjet propulsor. Three models (the rotor, the rotor-duct, and the PJP model) are established to separate the roles played by the rotor, the stator, and the duct in the vortex evolution of a PJP. In this paper, only the vortex structure of a single rotor is considered, with a discussion of the rotor-duct model and the PJP model being left to Part 2.

The remainder of the paper is organized as follows. In Sec. II, the numerical methodology is presented. In Sec. III, the numerical set-up is described, and the simulation results are validated by comparison with the experimental data. The results are presented and discussed Sec. IV from three main aspects: the open water coefficient and unsteady blade excitation force (Sec. IV A), vortex structure and evolution (Sec. IV B), and vortex dynamics (Sec. IV B). Finally, the conclusions are presented in $\mathrm{Sec} V$.

\section{NUMERICAL METHODOLOGY}

The governing equations are the Reynolds-averaged Navier-Stokes (RANS) equations, which are written as follows:

$$
\begin{aligned}
\frac{\partial u_{i}}{\partial x_{i}}= & 0 \\
\frac{\partial\left(\rho u_{i}\right)}{\partial t}+\frac{\partial\left(\rho u_{i} u_{j}\right)}{\partial x_{j}}= & -\frac{\partial p}{\partial x_{i}}+\frac{\partial}{\partial x_{j}}\left[\mu\left(\frac{\partial u_{i}}{\partial x_{j}}+\frac{\partial u_{j}}{\partial x_{i}}\right)\right] \\
& +\frac{\partial}{\partial x_{j}}\left(-\rho \overline{u_{i}^{\prime} u_{j}^{\prime}}\right)+S_{j}
\end{aligned}
$$

where $\rho$ is the fluid density, $x_{i}$ and $x_{j}(i, j=1,2,3)$ are the Cartesian coordinate components, $u_{i}$ and $u_{j}$ are the components of the absolute velocity in the inertial system, $p$ is the pressure, $t$ is the time, $\rho \overline{u_{i}^{\prime} u_{j}^{\prime}}$ are the Reynolds stresses, $S_{j}$ is a generalized source term, $\mu$ is the dynamic viscosity.

The improved delayed detached eddy simulation (IDDES) model ${ }^{?}$ is used in this paper to capture the structure of the vortices generated by the propeller. The IDDES model is based on a modification of the sink term in the $k$ equation of the shear stress transport (SST) model:

$$
\begin{aligned}
\frac{\partial(\rho k)}{\partial t}+\nabla \cdot(\bar{\rho} \mathbf{U} k)= & \nabla \cdot\left[\left(\mu+\frac{\mu_{T}}{\sigma_{k 3}}\right) \nabla k\right]+P_{k} \\
& -\rho \beta^{*} k \omega F_{\mathrm{IDDES}} \\
\frac{\partial(\rho \omega)}{\partial t}+\nabla \cdot(\bar{\rho} \mathbf{U} \omega)= & \nabla \cdot\left[\left(\mu+\frac{\mu_{T}}{\sigma_{k 3}}\right) \nabla \omega\right] \\
& +\left(1-F_{1}\right) 2 \rho \frac{\nabla k \cdot \nabla \omega}{\sigma_{\omega 2} \omega}+\alpha_{3} \frac{\omega}{k} P_{k} \\
& -\beta_{3} \rho \omega^{2}, \\
F_{\mathrm{IDDES}}= & \frac{l_{\mathrm{RANS}}}{l_{\mathrm{IDDES}}}
\end{aligned}
$$

where the parameter that defines this RANS model is the turbulence length scale $l_{\text {RANS }}=\sqrt{k} /\left(\beta^{*} \omega\right)$. The complete formulation is relatively complex, and more details can be found in Ref. ? .

\section{NUMERICAL SETUP}

\section{A. Geometry}

This paper considers a pre-swirl pumpjet propulsor for a large underwater robot whose design velocity is 20 knots. A 1:20 scale model is used for numerical work (as shown in Fig. 1(a)), which is the same as the water tunnel test model (see Fig. 1(b)). The coordinate system for the PJP is established as shown in Fig. 1(a). The coordinate origin is located in the geometric center of the rotor. The $\mathrm{Z}$-axis coincides with the rotation axis, and the positive direction is the same as the 


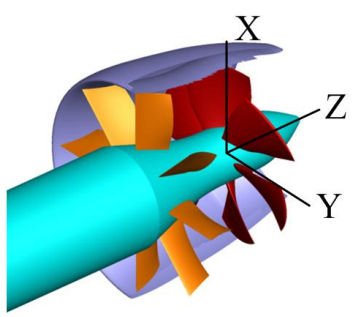

(a) The numercial model

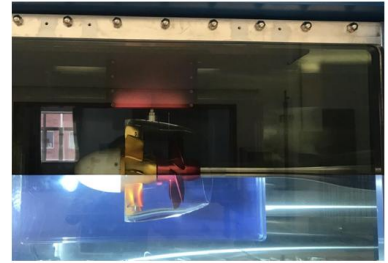

(b) The experiental model

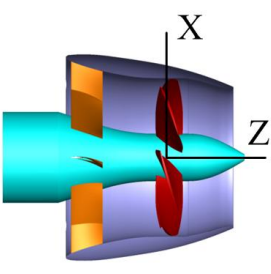

(c) Side view

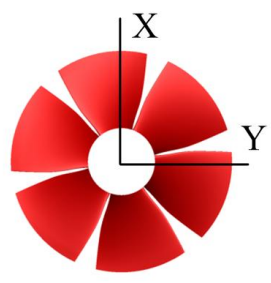

(d) rotor (front view)

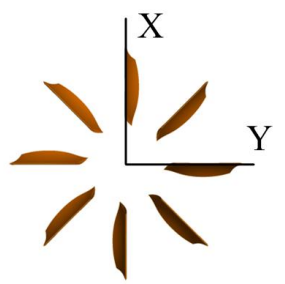

(e) stator (front view)

FIG. 1. The pre-swirl pumpjet propulsor (a) the numerical model; (b) the experimental model; (c) Side view of PJP; (d) Front view of the rotor; (e) Front view of the stator.

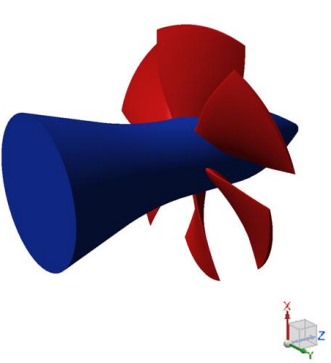

(a) Rotor only

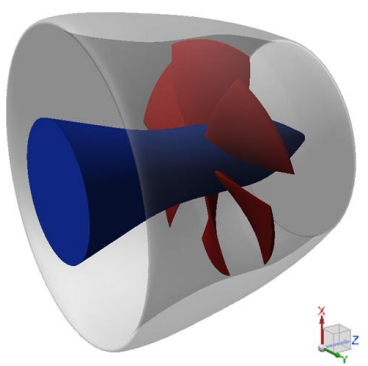

(b) Rotor-duct

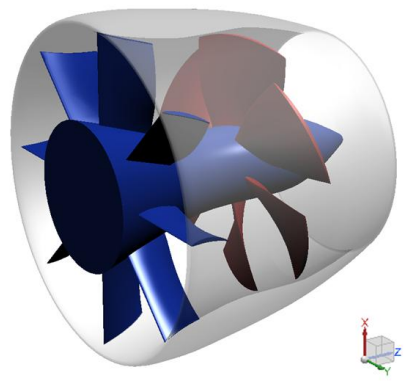

(c) PJP

FIG. 2. The three models: (a) rotor only (R model); (b) rotor-duct (R-D model); (c) PJP model. The rotor blades are shown in red and the stator blades in blue.

TABLE I. The geometric parameter of the duct.

\begin{tabular}{|c|c|c|c|c|c|c|c|c|c|c|c|}
\hline \multicolumn{12}{|c|}{ Dimensionless quantity of the duct section (the coordinate origin of propeller is at $z / D_{r}=0.64$ ) } \\
\hline$\overline{z / D_{r}}$ & 0 & 0.1 & 0.2 & 0.3 & 0.4 & 0.5 & 0.6 & 0.7 & 0.8 & 0.9 & 1.07 \\
\hline$y_{\text {inner }} / D_{r}$ & 0.6387 & 0.5868 & 0.5583 & 0.5359 & 0.5182 & 0.5077 & 0.5060 & 0.5060 & -0.4987 & 0.4762 & 0.4443 \\
\hline$y_{\text {outer }} / D_{r}$ & 0.6387 & 0.6590 & 0.6559 & 0.6444 & 0.6306 & 0.6151 & 0.5978 & 0.5759 & 0.5486 & 0.5166 & 0.4443 \\
\hline
\end{tabular}

TABLE II. The geometric parameter of the rotor and stator blades.

\begin{tabular}{lcccccrr}
\hline \hline \multirow{2}{*}{ Parameter } & \multirow{2}{*}{ Diameter } & $\begin{array}{c}\text { Blade number } \\
n\end{array}$ & $\begin{array}{c}\text { Pitch ratio } \\
P_{0.7} / D_{r}\end{array}$ & $\begin{array}{c}\text { Maximum thickness ratio } \\
t_{0.7} / D_{r}\end{array}$ & $\begin{array}{c}\text { Chord length ratio } \\
C_{0.7} / D_{r}\end{array}$ & Rake & Skew \\
\hline Rotor & 0.1664 & 6 & 4.3500 & 0.0218 & 0.3700 & 0 & 0 \\
Stator & 0.1930 & 8 & 0.8700 & 0.0168 & 0.2133 & 0 & 0 \\
\hline \hline
\end{tabular}

incoming flow direction. The X-axis is vertically upward direction. Finally, the Y-axis is determined by the right-hand rule. As shown in Fig. 1(c), for this pre-swirl pumpjet propulsor, the stator (see Fig. 1(e)) is located upstream of the rotor (see Fig. 1(d)), which can pre-whirl the inlet flow.

This PJP consists of six rotor blades $\left(N_{r}=6\right)$, eight stator blades $\left(N_{s}=8\right)$, and a duct. The mean diameter of the rotor blades is $D_{r}=0.1664 \mathrm{~m}$, the maximum diameter of the duct is $D_{d}=0.2200 \mathrm{~m}$, the length of the duct is $L_{d}=0.1762 \mathrm{~m}$, and the gap between the tips of the rotor blades and the duct is $1 \mathrm{~mm}$. Table I and Table II give the geometrical details of the pumpjet propulsor. In Table I, the $y_{\text {inner }}$ and $y_{\text {outer }}$ represent the $\mathrm{y}$ coordinates of the inner and outer surface of the duct section $(x=0)$, respectively. One thing should be mentioned here is that the installation position of the duct can be determined by the coordinate origin (the coordinate origin of propeller is located at $\left.z / D_{r}=0.64\right)$. Some main parameters of the rotor and stator blades are given in Table II. Among them, the pitch ratio, the maximum thickness ratio, and the chord length ratio use the corresponding value at $r=0.7 R$ ( $\mathrm{R}$ is the maximum radius of blade).

To analyze the influence of the stator and the duct on the evolution of the wake vortices of the rotor, three models are established: the R model [Fig. 2(a)], which has only a rotor and can be considered as a normal single propeller, the R-D model [Fig. 2(b)], which is composed of a rotor and a duct and 
is similar to a ducted propeller, and the PJP model [Fig. 2(c)].

To validate the CFD simulation results, a hydrodynamic experiment on this PJP model was carried out in the Water Tunnel of the Science and Technology on Water Jet Propulsion Laboratory, Marine Design and Research Institute of China (see Fig. 1(b)). The details of this experiment and the experimental data can be found in Ref. ?. The water tunnel has a speed range of $0-15 \mathrm{~m} / \mathrm{s}$, and the test conditions for this PJP were values of the advance coefficient $J$ in the range $0.2-1.2$, with a fixed rotational speed of $1200 \mathrm{rev} / \mathrm{min}$.

\section{B. Computational domains and meshes}

Here, only the flow of the $\mathrm{R}$ model is considered, with a discussion of the rotor-duct model and the PJP model being left to Part 2. Hence, only the computational domain of the $\mathrm{R}$ mdoel is shown here (see Fig. 3 (a)). The computational domain is a cylinder of diameter $10 D_{d}$ and length $20 D_{d}$. The whole computational domain is divided into three subdomains: the rotor domain, the near-field domain, and the farfield domain. The size of the near-field domain is a cylinder of diameter $1.5 \mathrm{D}$ and length $5 \mathrm{D}$ which is chosen according to the results of previous research. The subdomains are connected by interfaces through which flow simulation data are exchanged.

Structured grid of the $\mathrm{R}$ model is generated, and the details are shown in Fig. 3. Figure 3 (b) gives the meshes in the middle section ( $y-z$ plane) for the $\mathrm{R}$ model. The mesh in the wake of the propeller is refined to enhance the capture of wake vortices. More details of the mesh can be seen in Fig. 3 (c), where outer O-block grids are generated around the rotor blades and stator blades to enhance the capture of the boundary layer flow.

To validate the CFD simulation results (compared with the hydrodynamic experiment data of the PJP model), the grid of the PJP model is generated (see Figure 4). The topological structures of the R model and the PJP model are basically the same. Figure 4(b) shows the duct surface mesh. The mesh of slices of the rotor domain and the stator domain are shown in Figs. 4(c) and 4(d), respectively. For both grids, the mean $y^{+}$ value of the propeller surfaces is about 5 and the growth rate is 1.05 (the selection of $y^{+}$has been considered in a previous paper? ${ }^{?}$.

In the simulation, the rotational speed of the rotor is fixed as $1200 \mathrm{rev} / \mathrm{min}$, and the inlet velocity ranges from $0.67 \mathrm{~m} / \mathrm{s}-3.99 \mathrm{~m} / \mathrm{s}$ (with $\mathrm{J}$ ranges from $0.2-1.2$ ). Here, the Reynolds number of PJP is defined as $\mathrm{Re}=$ $\left[\rho C_{1.0 R} \sqrt{\left(\pi n D_{r}\right)^{2}+V_{\infty}^{2}}\right] / \mu$, where $C_{1.0 R}$ is the chord length of the rotor blade at $1.0 \mathrm{R}$ and $n$ is the rotational speed $(\mathrm{r} / \mathrm{s})$. and $\mu$ is the fluid dynamic viscosity. The Re of the PJP has a maximum value with about $8.5 \times 10^{5}$.

Table III lists the grid information. The mesh number of the rotor domain for the $\mathrm{R}$ model is $4.26 \times 10^{6}$ and those of the near-field and far-field domains are $4.44 \times 10^{6}$ and $0.65 \times 10^{6}$, respectively, giving a total mesh number of $9.35 \times 10^{6}$. The total mesh number is $12.53 \times 10^{6}$ for the PJP model.
TABLE III. Grid information for models.

\begin{tabular}{lccccr}
\hline \hline \multirow{2}{*}{ Mesh } & \multicolumn{5}{c}{ Total number $\left(\times 10^{6}\right)$} \\
\cline { 2 - 6 } & $\begin{array}{c}\text { Rotor } \\
\text { domain }\end{array}$ & $\begin{array}{c}\text { Stator } \\
\text { domain }\end{array}$ & $\begin{array}{c}\text { Near-field } \\
\text { domain }\end{array}$ & $\begin{array}{c}\text { Far-field } \\
\text { domain }\end{array}$ & Total \\
\hline R & 4.26 & - & 4.44 & 0.65 & 9.35 \\
PJP & 4.32 & 4.29 & 3.20 & 0.72 & 12.53 \\
\hline \hline
\end{tabular}

TABLE IV. Nondimensional physical quantities.

\begin{tabular}{lc}
\hline \hline Physical quantity & Definition \\
\hline Advance coefficient & $J=\frac{V}{n D_{r}}$ \\
Thrust coefficient of rotor & $K_{T r}=\frac{T_{r}}{\rho n^{2} D_{r}^{4}}$ \\
Torque coefficient of rotor & $K_{Q r}=\frac{Q_{r}}{\rho n^{2} D_{r}^{5}}$ \\
Torque coefficient of stator & $K_{Q s}=\frac{Q_{s}}{\rho n^{2} D_{r}^{5}}$ \\
Thrust coefficient of duct & $K_{T d}=\frac{T_{d}}{\rho n^{2} D_{r}^{4}}$ \\
Total thrust coefficient & $K_{T}=K_{T r}+K_{T d}$ \\
Total torque coefficient & $K_{Q}=K_{Q r}$ \\
Open water efficiency & $\eta=\frac{J}{2 \pi} \frac{K_{T}}{K_{Q}}$ \\
\hline \hline
\end{tabular}

\section{Validation}

Table IV lists the definitions of the nondimensional physical quantities used in the present study. In the table, $\rho$ is the fluid density, $n$ is the rotational speed of rotor, $D_{r}$ is the diameter of the rotor blades, $V$ is the far-field incoming flow velocity, $T_{r}$ and $T_{d}$ are the thrusts generated by the rotor and stator-duct, respectively ( $T_{d}=0$ for the $\mathrm{R}$ model), and $Q_{r}$ is the torque of the rotor.

The numerical simulation results for the PJP model for values of $J$ in the range 0.2-1.2 are compared with the experimental results in Fig. 5. For each value of $J, 45$ rotation cycles are calculated, and the average value of the last 30 cycles is taken as the final hydrodynamic performance prediction. As can be seen from Fig. 5, the results are in good agreement with the experimental values. The maximum relative errors $\Delta K_{T}$ and $\Delta K_{Q}$ are only $4.48 \%$ and $1.80 \%$, respectively for $J=0.2$. Considering the machining error of the actual experimental model, these numerical errors are acceptable. In a word, the hydrodynamic performance coefficient predicted by the IDDES simulation is accurate compared with the experiment data.

\section{Grid and time-step independence tests}

To carry out the grid independence test, three different grids are generated: a coarse grid, a medium grid, and a fine grid. 


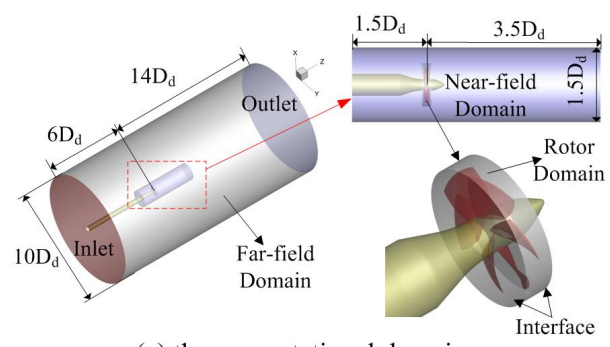

(a) the computational domain

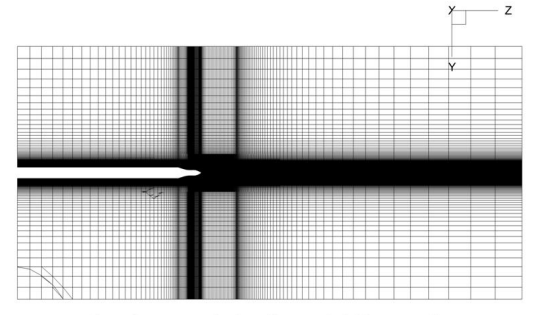

(b) the mesh in the middle section

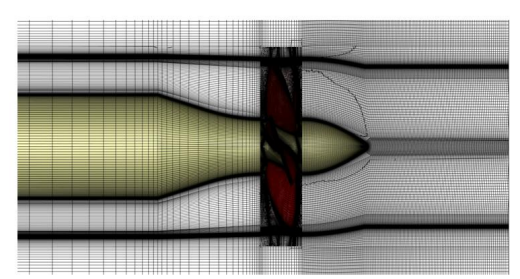

(c) the mesh around the rotor

FIG. 3. Computational domains and grids of the R model.

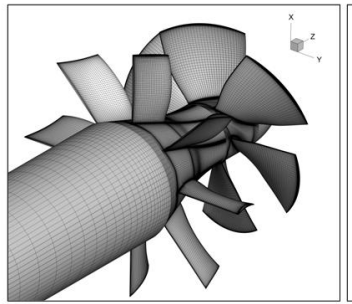

(a) Blades surface mesh

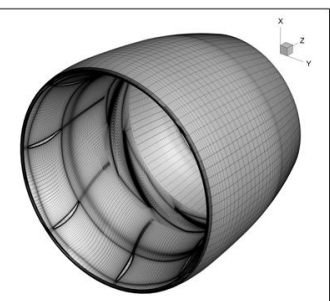

(b) The duct surface mesh

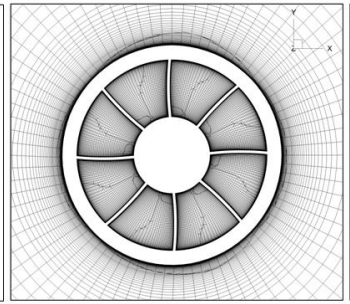

(c) Slice of the stator domain

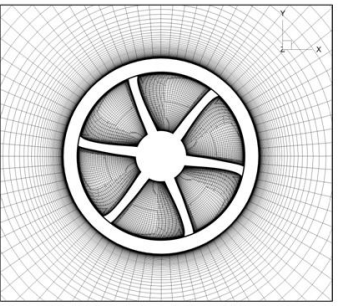

(d) Slice of the rotor domain

FIG. 4. Grid details for the PJP model: (a) blade surface mesh; (b) duct surface mesh; (c) slice of stator domain; (d) slice of rotor domain.

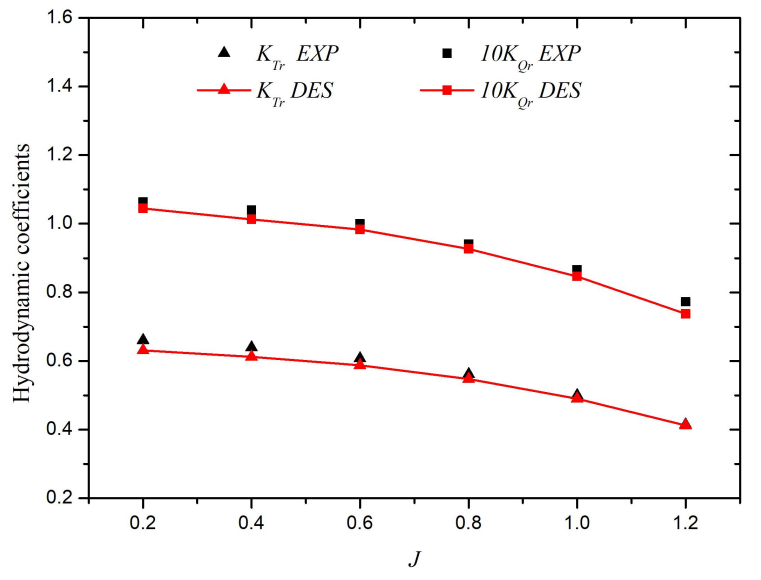

FIG. 5. Hydrodynamic coefficients of the PJP compared with experimental data.

Figure 6 compares the meshes in the $y-z$ plane of these three grids. For these three grids, the height of the first boundary layer mesh is kept unchanged to ensure that the $y^{+}$value near wall unchanged. However, the nodes in the axial, tangential and radial directions are encrypted. As can be seen, the grids inside the duct and in the wake region of the PJP model are gradually refined. The number of nodes distributed around the airfoil of the rotor for the coarse grid is 81 , and those of the medium and fine grids are 121 and 181, respectively. The maximum length of cells is defined as $\Delta_{\max }=\max \left(\Delta_{x}, \Delta_{y}, \Delta_{z}\right)$, where $\Delta_{x}, \Delta_{y}, \Delta_{z}$ is the length of cells in the $\mathrm{x}, \mathrm{y}$ and $\mathrm{z}$ direction, respectively. Considering the wake of PJP, we use the grid size at $1 D_{r}$ downstream of the duct as the judgment basis. For the coarse grid, the maximum size of cells at $1 D_{r}$ down-
TABLE V. Grid independence verification for the PJP model with the IDDES simulation.

\begin{tabular}{ccccccc}
\hline \hline$J$ & $\phi$ & Coarse & Medium & Fine & $e_{a}^{32}$ & $\mathrm{GCI}_{\text {medium }}^{32}$ \\
\hline \multirow{2}{*}{0.4} & $K_{T r}$ & 0.6146 & 0.6124 & 0.6122 & 0.0003 & $0.03 \%$ \\
& $K_{Q r}$ & 0.1020 & 0.1013 & 0.1016 & 0.0036 & $0.37 \%$ \\
& $K_{T r}$ & 0.4948 & 0.4903 & 0.4880 & 0.0048 & $0.49 \%$ \\
1.0 & $K_{Q r}$ & 0.0852 & 0.0847 & 0.0839 & 0.0094 & $0.96 \%$ \\
\hline \hline
\end{tabular}

stream of the duct is $\Delta_{\max }=2 \% D_{r}$, while $1.5 \% D_{r}$ and $1 \% D_{r}$ for the medium and fine grid. Similar discussions of the mesh refinement can be found in Ref. ? . The total number of cells for the coarse grid is $8.38 \times 10^{6}$, and those of the medium and fine grids are $12.53 \times 10^{6}$ and $24.59 \times 10^{6}$, respectively.

IDDES simulations of the three grids are carried out for $J=0.4$ and 1.0. Based on the theory of Celik et al.,? the grid convergence index (GCI) of the hydrodynamic coefficients for the PJP model is calculated, as shown in Table V. The numerical uncertainties in the medium-grid solution $\left(\mathrm{GCI}_{\text {medium }}^{32}\right)$ for $K_{T r}$ and $K_{Q r}$ have maximum values of about $0.96 \%$ for $J=1.0$. Hence the hydrodynamic performance exhibits good convergence as the grid is refined. In general, the grid independence is satisfactorily verified. Considering the computational time consumption, the medium grid is selected in the following simulations.

For time-step independence verification, three different time steps, $\Delta t=2 t, t$, and $0.5 t$ are chosen (where $t$ is the time taken for the rotor blades to rotate $1^{\circ}$ ). An IDDES simulation of the PJP model is performed for the case $J=1.0$. The results for the hydrodynamic coefficient of the PJP model at the different different time steps are compared in Table VI. As can be seen, both $K_{T r}$ and $K_{Q r}$ increase gradually and approach 


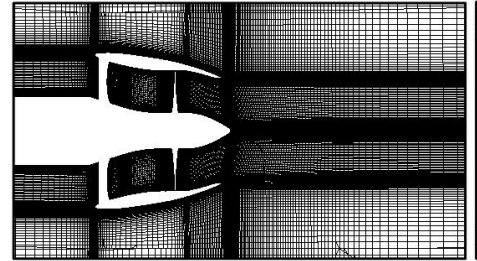

(a)

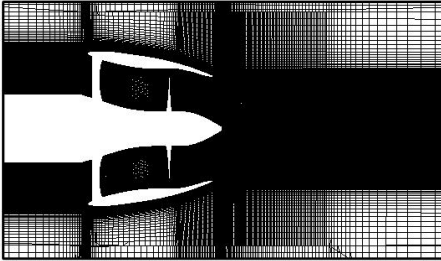

(b)

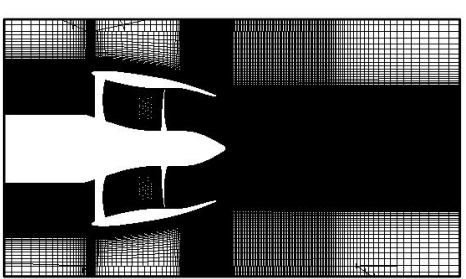

(c)

FIG. 6. Mesh details in the $y-z$ plane for (a) the coarse grid, (b) the medium grid, and (c) the fine grid.

TABLE VI. Time-step independence verification for the PJP model.

\begin{tabular}{lrrrc}
\hline \hline $\begin{array}{l}\text { Hydrodynamic } \\
\text { coefficients }\end{array}$ & \multicolumn{1}{c}{$2 t$} & \multicolumn{1}{c}{$t$} & \multicolumn{1}{c}{$0.5 t$} & EXP \\
\hline$K_{T r}$ & 0.4886 & 0.4903 & 0.4904 & 0.4973 \\
$K_{T s}$ & -0.0646 & -0.0658 & -0.0660 & - \\
$K_{Q r}$ & 0.0843 & 0.0846 & 0.0848 & 0.0866 \\
\hline \hline
\end{tabular}

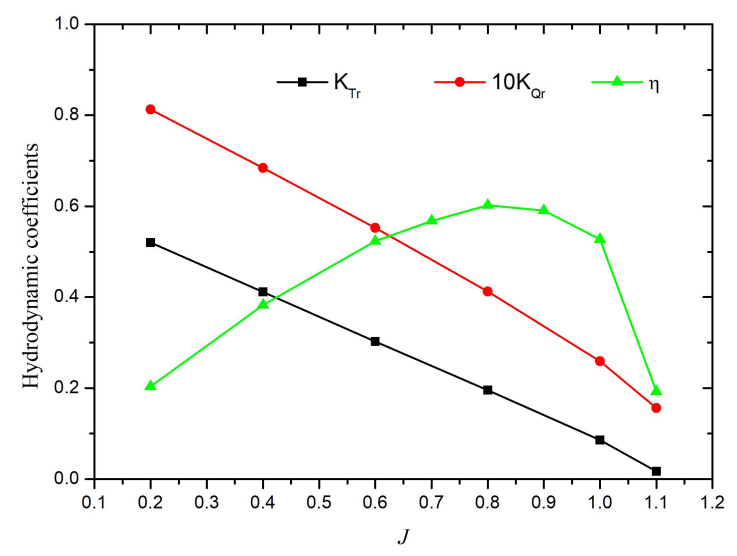

FIG. 7. Hydrodynamic performance of propellers: (a) open water coefficient; (b) $K_{T}$ and $10 K_{Q}$; (c) $K_{T r}$ and $10 K_{T d}$.

the experimental values as the time step decreases. Hence, the predicted hydrodynamic performance shows good convergence with changes in time step. Considering the rapid increase in calculational costs brought about by the use of very small time steps, we adopt $\Delta t=t$ in the following study.

Thus, both grid and time-step independence are satisfactorily verified.

\section{FLOW RESULTS AND DISCUSSION}

\section{A. Open water coefficient}

Figure 7 shows the hydrodynamic coefficients of the $R$ model. The $\mathrm{R}$ model, which is equivalent to an ordinary single propeller, reaches its maximum point about $60.24 \%$ for $J=0.8$. In addition, for the $\mathrm{R}$ model, both the thrust coefficient $K_{T r}$ and the torque coefficient $K_{Q r}$ decrease linearly with increasing $J$, whereas for the PJP model (see Fig. 5), it decreases with an increasing slope. It maybe caused by the presence of a stator. In addition, the $K_{T r}$ of the PJP has a significant increase at calculated cases of $J$ than the R model, which shows that the presence of a stator improves the working condition of the rotor very well. More details will be discussed in next part.

One thing should be mentioned here is that after exceeding the optimal efficiency point $(\mathrm{J}=0.8)$, the open water efficiency of the $\mathrm{R}$ model decreases sharply. This is a very important phenomenon because it will directly affect the instability of vortices of propeller. The relationship between vortices instability and $\mathbf{J}$ will be discussed in detail below.

\section{B. Vortex structure and evolution of the R model}

Figure 8 shows the instantaneous $Q$ surfaces of the R model for the case $J=0.8$. According to the experiment of Felli et $a l .{ }^{?}$ the wake vortex of an E779a propeller is mainly composed of a tip vortex and a hub vortex. Similar tip and hub vortices can be clearly observed in Fig. 8.

First, let us examine the tip vortex. For the $\mathrm{R}$ model, tip vortices are shed from the tip of each rotor blade and present themselves as a series of spiral filaments. As shown in Fig. 9(a), for $J=0.8$, these filaments maintain their spiral shape for a long distance downstream of the propeller until they gradually break down and disappear in the far field.

Second, from Fig. 8(b), we can see that the hub vortex falls off from the tail of the hub and then retains a straightline shape until the far field. Figure 9(b) shows the threedimensional structure of the hub vortex. As can be seen, the hub vortex is not completely stable, but undergoes spiral precession, which is similar to the picture captured by Felli et $a l .{ }^{?}$ in their experiment. The rotational frequency of the hub vortex will be discussed in the later dynamic analysis.

Figure 10 shows the complex vortex system of the $\mathrm{R}$ model, with the vortices indicated by red and black circles. As well as the hub and tip vortices, three series of small vortices called the trailing tip vortex, trailing root vortex 1 and trailing root vortex 2 , are generated. In fact, the trailing tip vortex and trailing root vortices 1 and 2 originate from the trailing edge vortex sheet of the rotor blade. According to the work by Okulov and Sørensen,? the trailing edge vortex is a helical vortex sheet that is shed from the trailing edge of the rotor blade as a result of the nonconstant circulation of the rotor. These trailing edge 


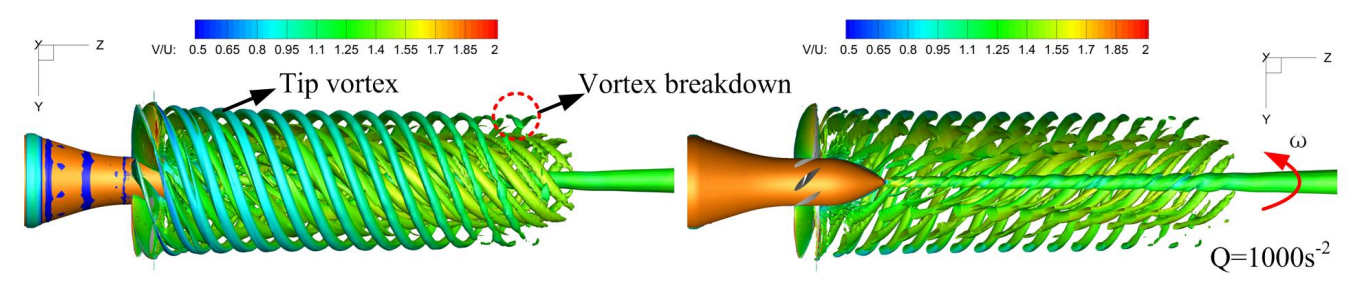

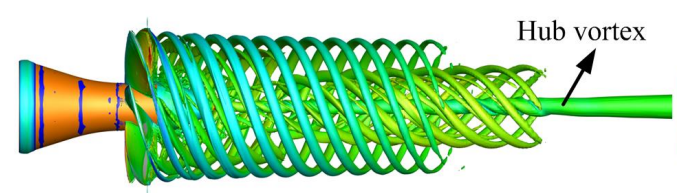

(a)

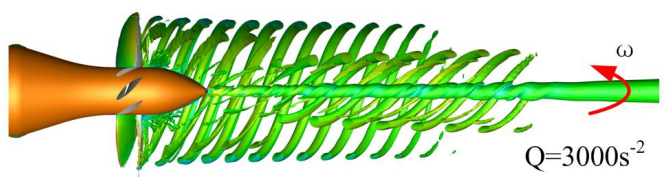

(b)

FIG. 8. Instantaneous $Q$ surfaces of the R model for the case $J=0.8$, colored by the velocity magnitude scaled by $U$ : (top) $Q=1000 \mathrm{~s}^{-2}$; (bottom) $Q=3000 \mathrm{~s}^{-2}$. (a) Overall view. (b) View of the half-model.

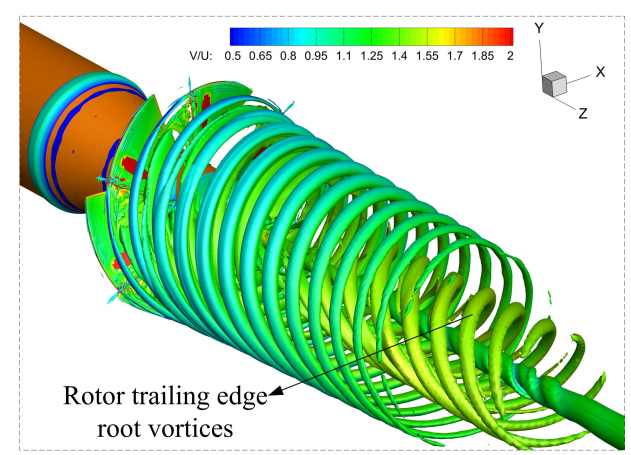

(a) $\mathrm{Q}=3000 \mathrm{~s}^{-2}$

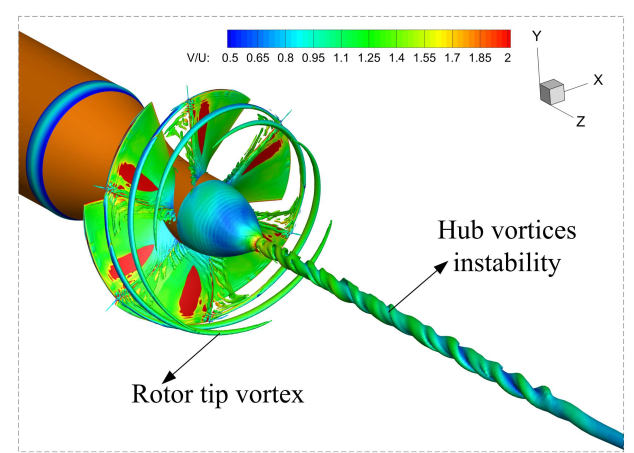

(b) $\mathrm{Q}=10000 \mathrm{~s}^{-2}$

FIG. 9. Three-dimensional views of the vortex structure of the R model for $J=0.8$ : (a) instantaneous $Q$ surface $\left(Q=3000 \mathrm{~s}^{-2}\right)$; (b) instantaneous $Q$ surface $\left(Q=100000 \mathrm{~s}^{-2}\right)$.

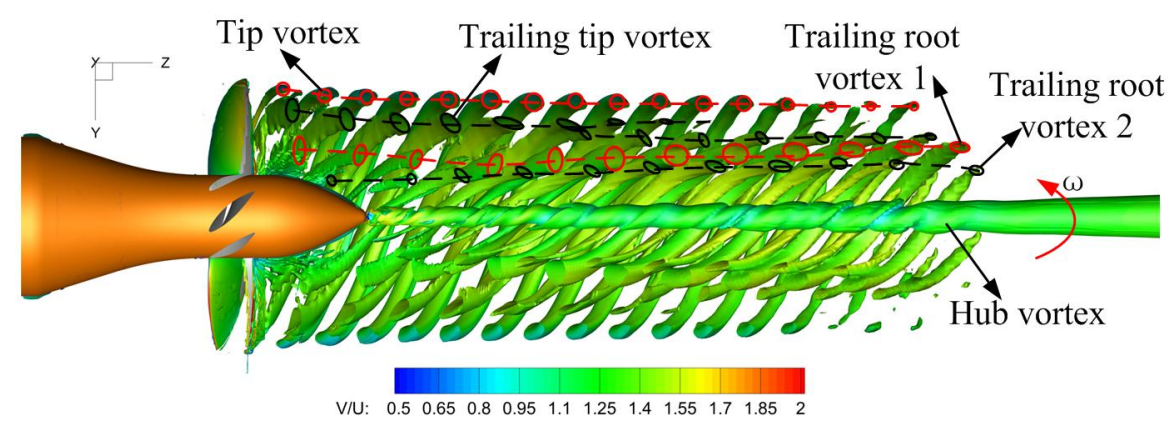

FIG. 10. Vortex system of the R model shown as instantaneous $Q$ surfaces $\left(Q=1000 \mathrm{~s}^{-2}\right)$.

vortices and strong tip vortices form a screw surface behind the rotor.

Figure 11 shows a three-dimensional view of the structure of the trailing edge vortex sheet of the rotor. As can be seen from Fig.11(a), a layer of the vortex sheet falls off from the edge of the rotor, and then gradually collapses along with the strong tip vortices. At the tip and root of the trailing edge, two groups of strong vortices [see Fig. 11(b)] are formed, which are called trailing tip and trailing root vortices, respectively.

With the development of trailing tip vortices downstream, a group of spiral filaments are formed on the inner side of the rotor tip vortices (see Fig. 10). The spiral direction of the trailing tip vortex filaments is the same as that of the rotor tip vortices.

The trailing root vortices are shed from the root of the rotor blade trailing edge and consist of small vortices [as shown in 


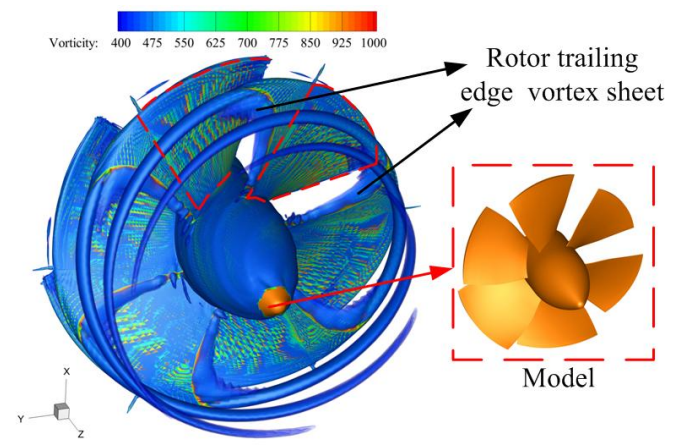

(a)

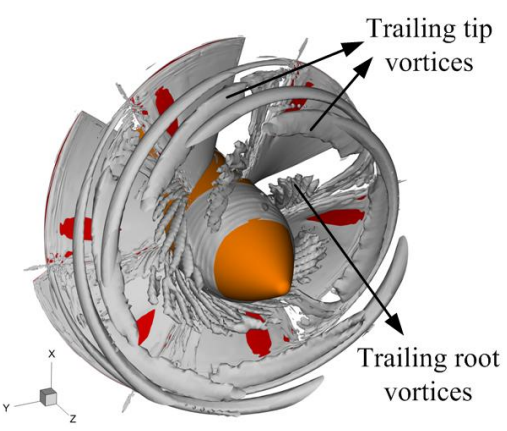

(b) $\mathrm{z} / \mathrm{D}<0.3$

FIG. 11. Views of the trailing edge vortex sheet: (a) instantaneous surfaces of vorticity magnitude; (b) instantaneous $Q$ surface $\left(Q=5000 \mathrm{~s}^{-2}\right)$.

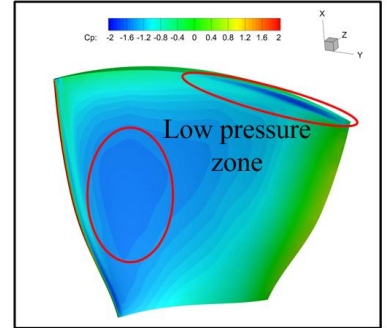

(a)

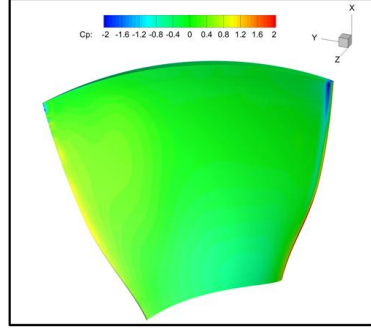

(b)

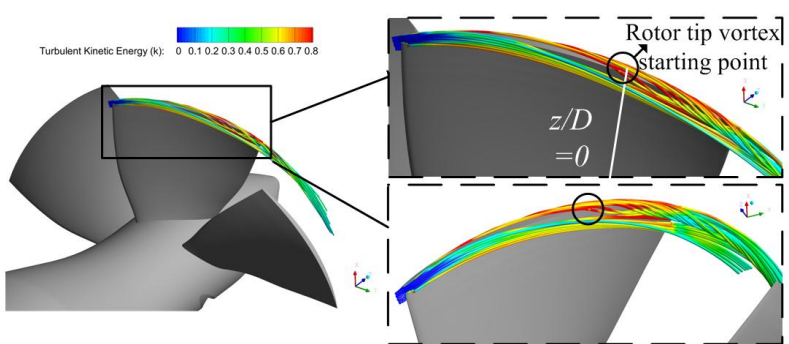

(c)

FIG. 12. Views of tip vortices: (a) contours of the pressure coefficient $C_{p}$ on the suction side of the rotor; (b) contours of $C_{p}$ on the pressure side of the rotor; (c) streamlines at the tip of the rotor blade.

Fig. 11(b)]. With the development of the trailing root vortices downstream, two groups of spiral filaments are formed around the hub vortex, which are called the trailing root vortex 1 and trailing root vortex 2 , respectively. They rotate in the same direction as the rotor tip vortex.

Similar trailing root vortex structures have been found in other studies. ? As shown in Fig. 10, the diameter of the root vortex gradually increases with its downstream development. In the far-field region, the diameter is about $0.5 D_{r}$. In addition, the trailing root vortex survives for a greater distance from the rotor than the trailing tip vortex, which was also found by Posa et al.

For the $\mathrm{R}$ model studied here, we speculate that the formation of the trailing tip vortex is due to the unique blade with a broad tip designed for duct propellers and PJPs. The E779a propeller studied in the experiments by Felli et al. ${ }^{\text {? }}$ and Posa et $a l^{?}$ is a typical single propeller with sharp-tipped blades. Therefore, the trailing tip vortices of E779a merge with the rotor tip vortices, resulting in the disappearance of the trailing tip vortices. Figure 12 confirms this conjecture. Figures 12(a) and 12(b) show contours of the pressure coefficient of a rotor blade, and it can be seen that there are two obvious lowpressure areas on the suction side of the blade, while the pressure in most areas on the pressure side is higher, resulting in fluid being sucked from the pressure side to the suction side, which eventually leads to the formation of a rotor tip vortex.

We can see that a tip vortex forms from about $z / D=0$ (about the middle of the rotor) and then gradually sheds from the tip of the rotor. Therefore, the tip vortex is not shed from the trailing edge of the rotor blade, as in the case of an ordinary single propeller, but rather falls off in advance at the tip before the trailing edge. Hence, the trailing tip vortex is formed owing to premature shedding of tip vortices by the $\mathrm{R}$ model compared with a normal single propeller.

Figure 13 shows the instantaneous flow field in the $y-z$ plane of the R model for $J=0.8$. From the contours of the pressure coefficient in the $y-z$ plane in Fig. 13(a), the positions of the tip vortices can be clearly observed as low-pressure zones marked by red circles. As can be seen, after shedding from the rotor blade $\left(r=0.5 D_{r}\right)$, the tip vortices gradually shrink inward in the radial direction until $z / D=1.5$, after which they remain nearly unchanged at about $r=0.4 D_{r}$ and $z / D=1.5-3$. After $z / D=3$, the low-pressure region of the tip vortex gradually merges with the low-pressure wake behind the propeller and finally dissipates in the far field. The hub vortex appears as a straight low-pressure area after shedding from the tail of the hub from $z / D=0.5$ to the far field.

The axial velocity in the $y-z$ plane is visualized in Fig. 13(b), and the periodic fluid acceleration caused by the rotor blade rotation can be observed. Figure 13(c) shows the vorticity magnitude in the $y-z$ plane, and, in addition to conspicuous areas of tip and hub vortices, there is an obvious wake caused by the trailing edge vortex and persisting for a long distance (from $z / D=0$ to 2.5). Hence, the near field downstream of the propeller is dominated by coherent tip vortices, a concentrated hub vortex, and the blade trailing edge 


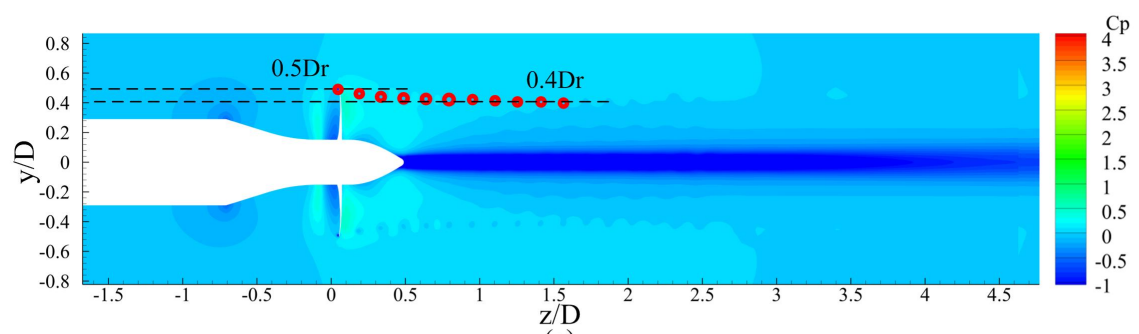

(a)
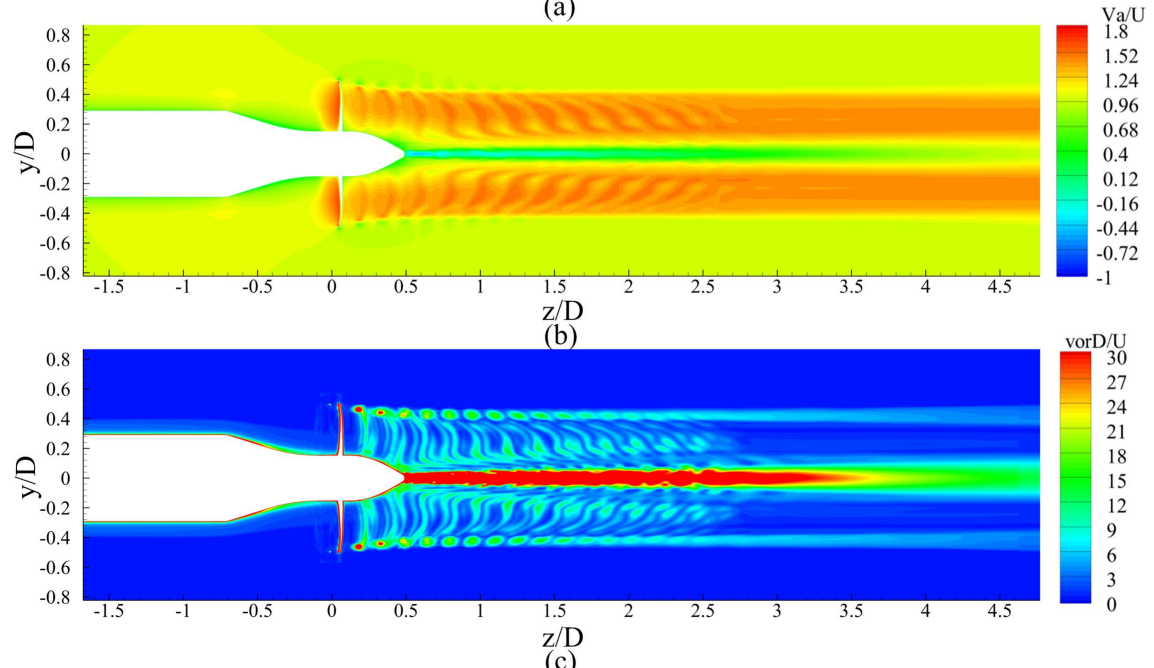

(c)

FIG. 13. Instantaneous flow field in the $y-z$ plane of the R model for $J=0.8$ : (a) pressure coefficient; (b) axial velocity; (c) vorticity magnitude. The axial velocity is scaled by $U$, and the vorticity magnitude is normalized with $U / D$.

wake.

According to Okulov and Sørensen, ${ }^{?}$ a complicated roll-up process occurs owing to the mutual interaction between the trailing edge vortices and the tip vortices. More importantly, the roll-up process will have a significant effect on the stability of the tip vortices.

Instantaneous surfaces of the vorticity magnitude are shown in Fig. 14(a), while Fig. 14(b) shows the contours of the $x$ component of vorticity in the $y-z$ plane. The axial evolution of the blade trailing vortex sheet can be clearly observed. Based on these two figures, we show in Fig. 14(c) a schematic representation of the roll-up process of the rotor blade trailing edge vortex sheet in the $y-z$ plane. In this illustration, different colored ovals represent vortex centers with different directions of rotation $\left(\omega_{x}\right)$. Blue means that the $x$ component of vorticity is negative, while red means that it is positive, and the corresponding rotation direction is indicated with an arrow. As previously discussed, owing to the premature shedding of tip vortices, the trailing tip vortices will separate from those tip vortices after falling off the tip of the blade, as shown by the rotor tip vortices and trailing tip vortices in Fig. 14(c) (marked with blue ovals).

The spatial evolution of the roll-up of the rotor blade trailing edge vortex is reflected not only in the axial direction (Fig. 14), but also in the radial direction (Fig. 15). The contours of the vorticity magnitude at different $z$ positions are shown in Fig. 15.

After shedding from the trailing edge of the rotor, the vor- tex has the same shape as the trailing edge. Then, as it develops downstream (from $z / D=0.1$ ), the upper part of the trailing vortex gradually bends. For greater clarity, we give a schematic representation of the roll-up process in Fig. 16. This progressive bending of the trailing edge vortex is called roll-up. More specifically, the trailing edge vortex gradually becomes distorted, with progressively increasing angular displacements and a radial rise of its upper part. As a result, the distance between the trailing edge vortex and the next tip vortex gradually decreases, which means that the mutual interaction between the trailing edge vortex and the tip vortex becomes stronger and stronger with downstream development. This process is similar to the phenomenon described by $\mathrm{Ku}-$ mar and Mahesh.?

We can see that with increasing $z / D$, the trailing wake gradually diverges from the shape of the trailing edge $(z / D=0.1-$ $0.2)$ and divides into several separate wakes $(z / D=0.3-1)$. At $z / D=0.4$ [see Fig. 15(f)], the wake of the former trailing vortex is in contact with the next tip vortex. After that, the interaction between the tip vortex and the corresponding trailing vortex becomes weaker and weaker. At $z / D=1$, the two vortices are almost completely separated. Therefore, the rollup process can be divided into the following three processes: (1) the top of the trailing edge vortex bends $(z / D=0.1-0.3)$; (2) the upper part of the trailing edge vortex approaches and comes into contact with the next tip vortex $(z / D=0.4-0.6)$; (3) there is complete separation of the trailing edge vortex from the previous tip vortex $(z / D=0.6-1.0)$. If we adopt po- 


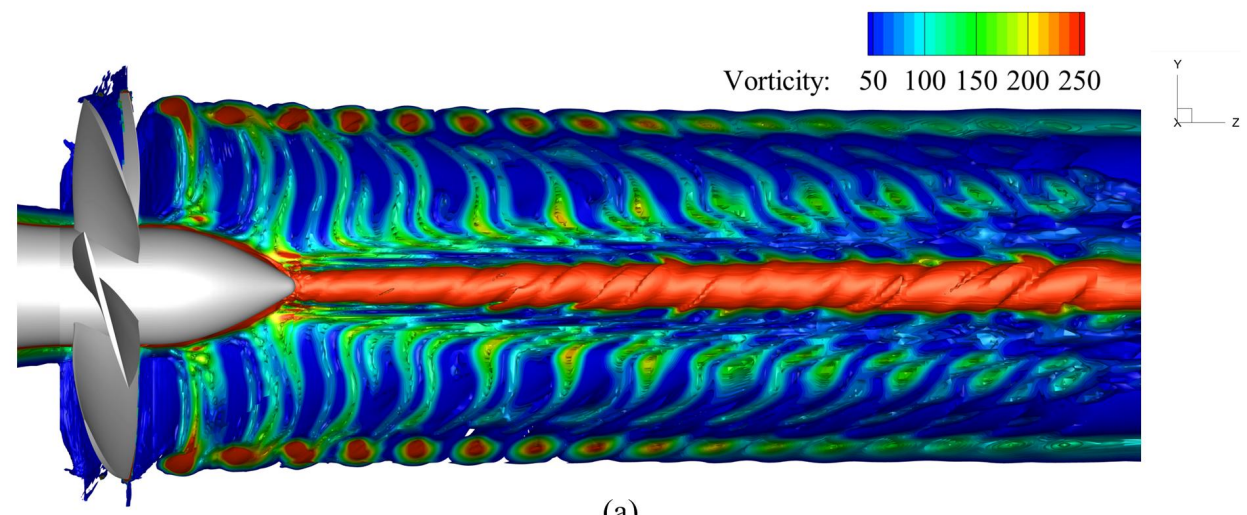

(a)

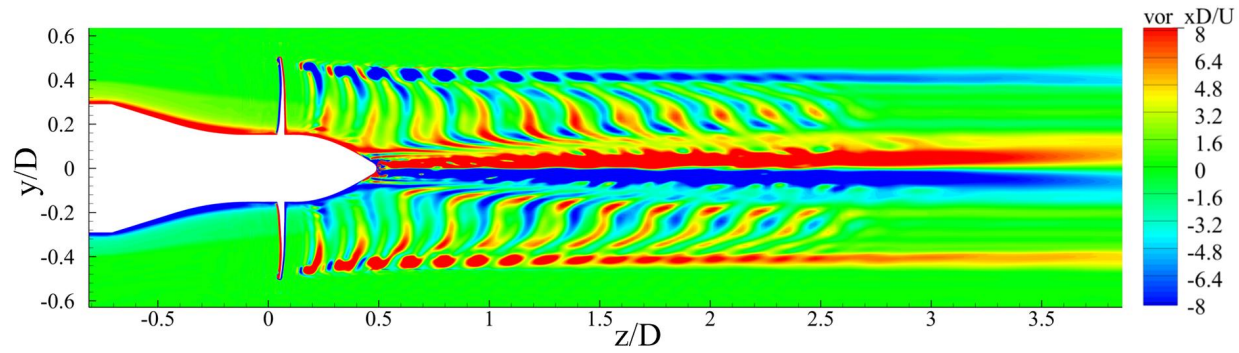

(b)

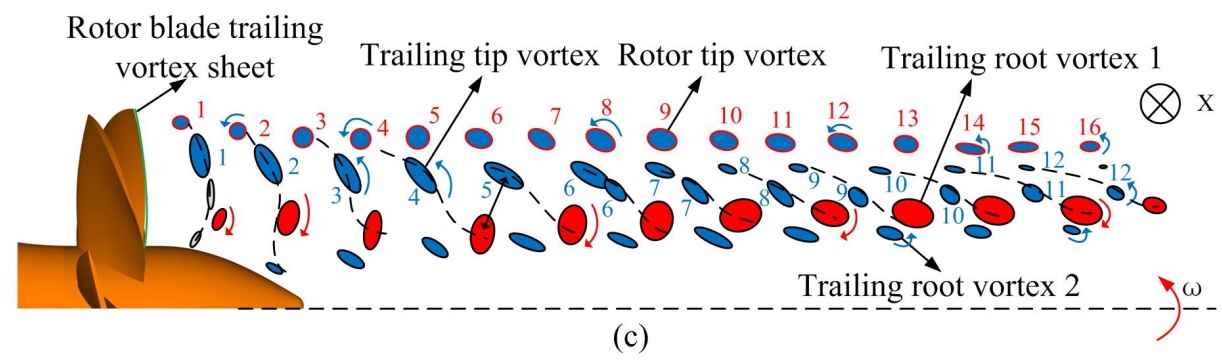

FIG. 14. Axial evolution of the blade trailing edge vortex sheet of the R model for $J=0.8$ : (a) instantaneous surfaces of vorticity magnitude scaled by $U / D$; (b) The $x$ component of vorticity in the $y-z$ plane normalized with $U / D$; (c) schematic representation of rotor blade trailing edge vortex sheet roll-up in the $y-z$ plane.

lar coordinates in the $x-y$ plane (radius $r$ and angle $\varphi$ ), then the phase difference between the tip vortex and the corresponding trailing vortex is small at at $z / D=0.1$ but becomes larger and larger with increasing $z$. At $z / D=1$, the trailing vortex has a phase lag of about $60^{\circ}$ compared with the corresponding tip vortex.

This roll-up process takes place in three dimensions, and its axial evolution is shown in Fig. 14(c). From $z / D=0.1$ to 0.6 , corresponding to the roll-up of the trailing vortex, the pitch of the trailing tip vortex increases gradually (note the closeness of the third trailing tip vortex to the fourth tip vortex.).

According to Okulov's conjecture, ${ }^{?}$ the complete development of the roll-up process triggers instability of the tip vortices. However, according to the present results, for the $\mathrm{R}$ model, the behavior is entirely different. After the complete development of the roll-up process (at nearly $z / D=0.6$ ), the tip vortex always remains stable, with a fixed radius and the same spiral-to-spiral distance. The conclusion that complete development of the roll-up process triggers instability of the tip vortices does not seem to apply to this model.

We suspect that, as discussed before, for the present $\mathrm{R}$ model, the separation of the tip vortex and the trailing tip vortex caused by the wide tip blade is the reason. It should be mentioned that the directions of rotation of the tip vortices and the trailing tip vortices are the same [with the same vorticity $x$ components shown as blue ovals in Fig. 14(c)]. Therefore, it seems that there is a repulsive force between the tip vortex and trailing tip vortex, which causes a significant decrease in the radius of the trailing tip vortex, while the tip vortex radius remains nearly unchanged. The trailing tip vortex takes the place of the tip vortex. After the full development of the roll-up process (from $z / D=0.6$ ), the tip vortex and the trailing vortex are completely separated, as a consequence of which the interaction between the trailing root vortex and the tip vortex is replaced by that between the trailing root vortex and the trailing tip vortex. Figures 14(b) and 14(c) support this conclusion. The directions of rotation of the trailing root vortex and the trailing tip vortex are opposite, and so there is 


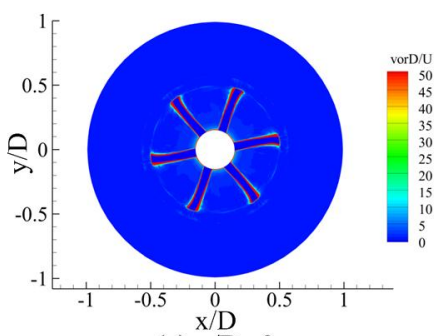

(a) $\mathrm{z} / \mathrm{D}=0$

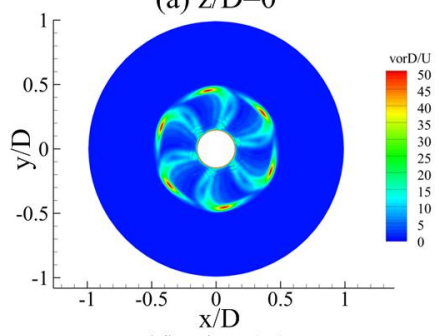

(d) $\mathrm{z} / \mathrm{D}=0.2$

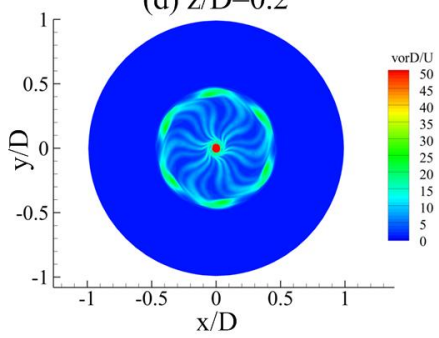

(g) $\mathrm{z} / \mathrm{D}=0.5$

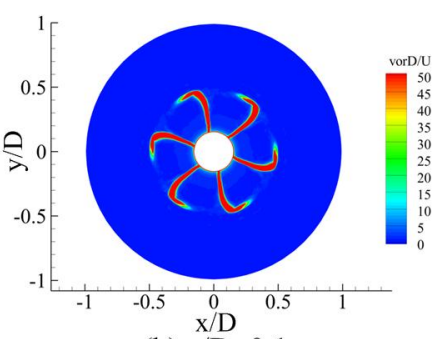

(b) $\mathrm{z} / \mathrm{D}=0.1$

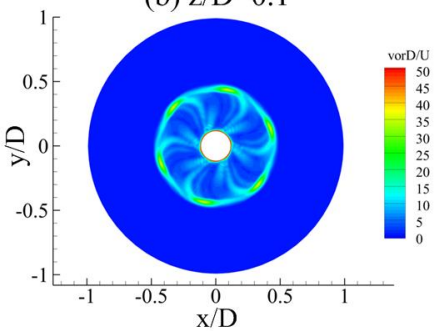

(e) $\mathrm{z} / \mathrm{D}=0.3$

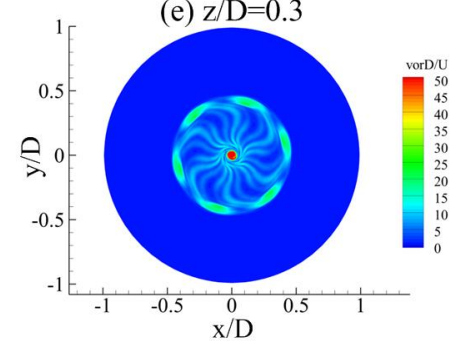

(h) $\mathrm{z} / \mathrm{D}=0.6$

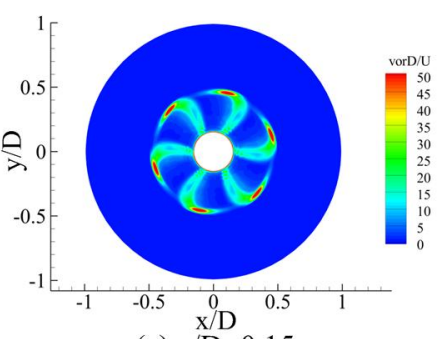

(c) $\mathrm{z} / \mathrm{D}=0.15$

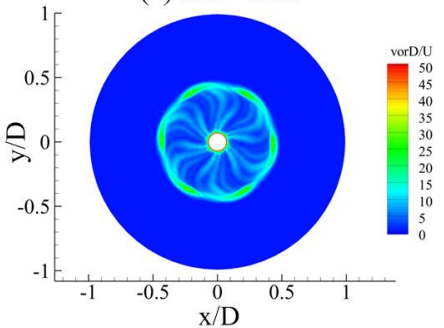

(f) $\mathrm{z} / \mathrm{D}=0.4$

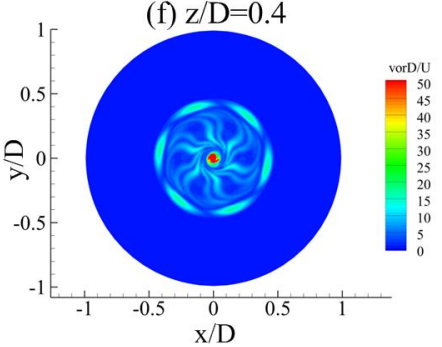

(i) $\mathrm{z} / \mathrm{D}=1$

FIG. 15. Contours of the vorticity magnitude in the $x-y$ plane as the flow develops downstream (increasing $z / D$ ) for $J=0.8$, normalized with $U / D$.
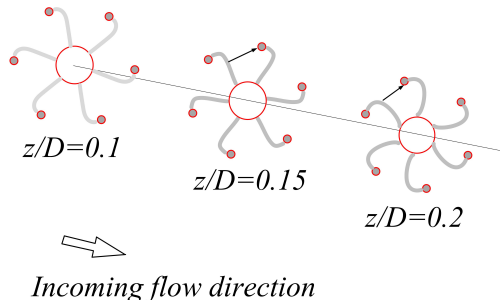

Incoming flow direction

FIG. 16. Schematic diagram showing rotor blade trailing edge vortex sheet roll-up in the $y-z$ plane, $J=0.8$.

mutual attraction between these two vortices, resulting in an increase in the radius of the trailing root vortex (which is the same conclusion as that drawn by Kumar and Mahesh? ${ }^{\text {) }}$. At $z / D=1.5$, the trailing tip vortex splits into two parts owing to the attraction by the trailing root vortex. After that, the main part is attracted to the trailing root vortex and finally fused with it, while the remaining small part gradually dissipates [see Fig. 14(a)].

In conclusion, for the $\mathrm{R}$ model, for $J=0.8$, the premature shedding of tip vortices leads to the appearance of a trailing tip vortex. This trailing tip vortex takes over the role of the tip vortices, which leads to the disappear of the mutual interaction of two adjacent tip vortices (i.e., without the occurrence of the mutual-inductance instability mode? ${ }^{?}$ ), as a consequence of which the tip vortices are stable for the $\mathrm{R}$ model.

The hub vortex of the $\mathrm{R}$ model is different from that of the E779a propeller. In the experiment by Felli et al.,? the hub vortex remained stable and started to oscillate, following a spiral shape from the transition point until double-helix breakdown occurred in the far field. For the R model, the hub vortex takes the form of twisted vortices until it fades out into a large vortex in the far field (see Fig. 10). It should be noted that the region in which the hub vortex maintains its twist is basically the same as the region in which trailing root vortices exist (see Fig. 10). When the trailing root vortices gradually dissipate, the distortion of the hub vortex gradually disappears. Therefore, we can reasonably speculate that the existence of trailing root vortices is the reason why the hub vortex can continue to twist without the occurrence of instability.

The mechanism by which instability of the tip and hub vortices is triggered will be discussed below.

Figure 17 shows the instantaneous $Q$ surface of the $\mathrm{R}$ model for different values of $J$. As classified by Widnall? ${ }^{?}$ and Felli et al., ${ }^{?}$ there are three main instability modes of a tip vortex: short-wave instability, long-wave instability, and mutualinductance instability.

Here, the evolution of the tip vortices of the R model ex- 
hibits different instability modes for different values of $J$. As defined by Widnall, ${ }^{?}$ the mutual-inductance instability mode occurs when adjacent helical tip filaments begin to interact strongly. At low $J(J=0.2,0.4$, and 0.6$)$, twisting of tip vortices and contact between adjacent vortices can be clearly observed. According to Felli et al.,? the instability inception point of tip vortices is defined as the first position at which the gradient of the tip vortex envelope equals $50 \%$ of the maximum slope. Here, it is hard to adopt their definition, since the maximum slope of the tip vortex envelope can be zero in some cases, such as $J=0.8$. Hence, we give a relatively vague definition of the instability inception point of tip vortices as the first position at which the tip vortex is becoming destabilized. More specifically, the filament of the tip vortex begins to twist and the shape of the filament is no longer a standard spiral. According to the instability inception point, the wake of the R model can be divided into two parts: a stable region and an unstable region. The instability inception points of tip vortices for different values of $J$ are shown by the dashed lines in Fig. 17(a). For $J=0.8$, there is an instability inception point at which short-wave instability is observed. According to Saffman,? the short-wave instability shows as a smooth-sinuous-wave-type mode, with small sinusoidal displacements of one filament (see Fig. 19). For $J=1.0$, an instability inception point appears at which the tip vortices break down.

To quantitatively study the relationship between spiral-tospiral distance and tip vortex instability, the helical pitch of tip filaments, $P$, is defined as the axial distance between two adjacent helical filaments. The axial distance that a spiral filament moves in one revolution is called the lead $S$. Thus, $S=n P$, where $n$ is the number of blades on the rotor ( $n=6$ here).

It should be noted that $P$ changes as the tip vortices evolve, especially in the case $J=0.2$, for which violent interactions between adjacent tip filaments occur. The instantaneous helical pitch ratio of tip filaments, $P / D$, for different values of $J$ is shown in Fig. 18. According to these results, the $P / D$ between tip filaments 1 and 2 increases monotonically with increasing $J$. For $J=0.2, P / D$ is about 0.1 , while it is about 0.15 for $J=1.0$.

To show the instability of the tip vortex more clearly, the instantaneous three-dimensional trajectories of a single filament for the R model at different values of $J$ are shown in Fig. 19.

For $0.2<J<0.8$ (below the optimal operating point for the $\mathrm{R}$ model), $P / D$ of the first two tip filaments increases monotonically with increasing $J$ (see Fig. 18), and, similarly, the instability inception point moves further upstream with increasing $J$ (see Fig. 19, where the instability inception point moves from $z / D=0.4$ to 1.7 as $J$ increases from 0.2 to 0.8 ). Therefore, it can be concluded that the instability of the tip vortex depends on helical the pitch ratio of tip filaments, $P / D$. This is consistent with the conclusions of Felli et al. ${ }^{?}$ and Okulov? that the transition to instability of the tip vortex shows a clear dependence on the spiral-to-spiral distance. More specifically, the larger the value of $P / D$, the weaker is the interaction between adjacent tip filaments, the less likely is the mutualinductance instability mode to occur, and the longer is the stable length of tip vortices.
In the case $J=1.0$, as shown in Fig. 17, the tip vortex dissipates rapidly after shedding from the tip, owing to the rotor working in an unfavorable condition. It is therefore hard to define an instability inception point. Here, we take the instability inception point as being the point at which the tip vortex begins to break down. Compared with the case $J=0.8$, the $P / D$ of the tip vortex for $J=1.0$ increases slightly, while its stable length drops sharply. This sharp decrease in the stable length of the tip vortex is related to the decrease in its strength, rather than to interaction between tip vortices. Support for this speculation comes from the fact that (see Fig. 17) for $J=0.8$ and 1.0 , there is no mutual-inductance instability, but breakdown gradually occurs during transition of the tip vortex from the stable to the unstable region.

For low $J$ (in the range $0.2-0.6$, especially $J=0.2$ ), the mutual-inductance instability mode clearly occurs. For the $\mathrm{R}$ model, the mutual-inductance instability mode for tip vortices is quite different from the classic "leapfrogging" phenomenon. More information about the leapfrogging phenomenon can be found in Ref. ?

A detailed illustration of this unique instability mode for the $\mathrm{R}$ model is presented in Fig. 20:

(i) During $t_{0}$ to $t_{0}+\frac{1}{8} T$, filaments 1 and 2 begin with the same spiral shape. During this period, the filaments have just detached from the tip of the rotor, the space between filaments is uniform, and there is as yet no mutual interaction between them.

(ii) At $t_{0}+\frac{1}{4} T$, as a result of the mutual inductance between adjacent filaments, filament 2 is pulled closer to filament 1 . It should be mentioned that the radius of filament 2 decreases as it moves [see Fig. 20(d)]; meanwhile, the radius of filament 1 (the front filament) expands slightly (similar to the leapfrogging phenomenon).

(iii) During $t_{0}+\frac{3}{8} T$ to $t_{0}+\frac{1}{2} T$, filament 2 comes into contact with filament 1 (with the radius of filament 2 being smaller than that of filament 1). Filament 2 is then pulled forward through filament 1 (the front filament), resulting in overlapping of these filaments. This phenomenon is slightly different from what Felli et al. ${ }^{?}$ observed in their experiment, where filament 2 kept moving downstream after it was pulled forward through filament 1 . Here, after filament 2 is pulled before filament 1 , it is pulled back to filament 3 again. Filament 2 is attracted by filament 3 , forming a new filament $2-3$ overlap.

(iv) During $t_{0}+\frac{5}{8} T$ to $t_{0}+\frac{7}{8} T$, the same process is repeated: filament 4 moves close to filament 3 , and these filaments then come into contact and overlap. Finally, filament 5 merges with filament 6 .

Processes (i)-(iv) represent the time evolution of the tip vortex for the R model. This mutual inductance instability of the tip vortex is called the "overlap-forward" phenomenon here.

Next, we consider the spatial evolution of the tip vortex. Figure 21 shows the three-dimensional vortex structure at $t=$ 


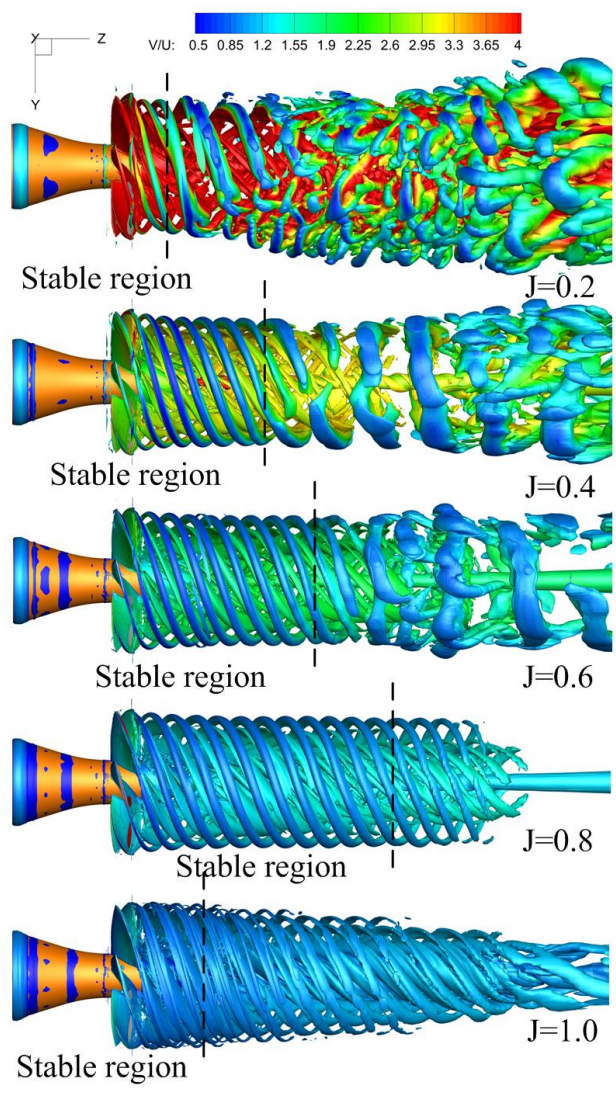

(a) $\mathrm{Q}=1000 \mathrm{~s}^{-2}$
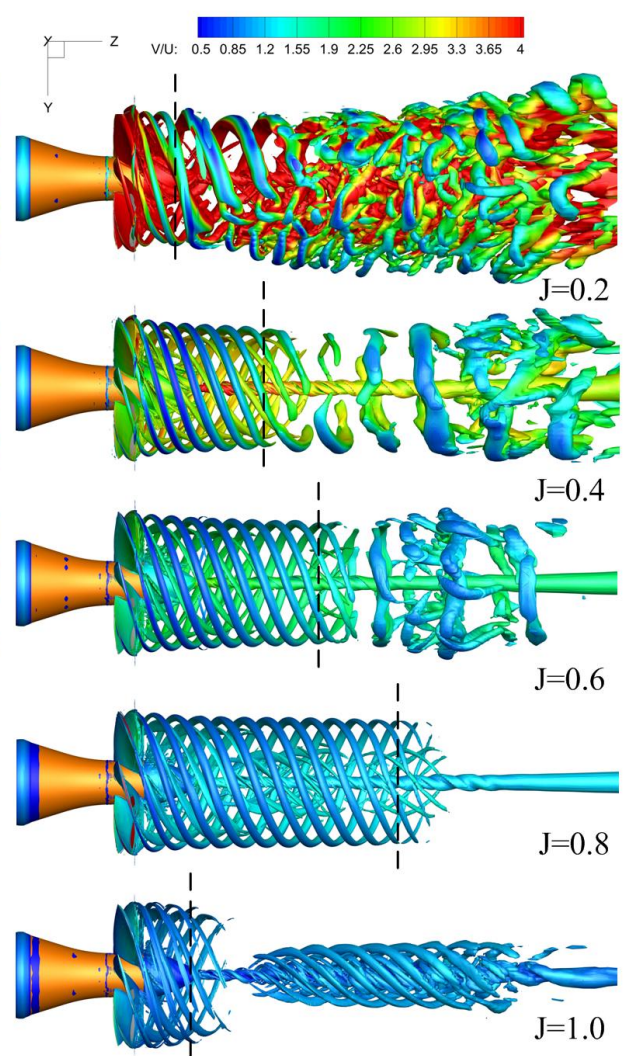

(b) $\mathrm{Q}=5000 \mathrm{~s}^{-2}$

FIG. 17. Instantaneous $Q$ surfaces of the R model for different values of $J$, colored by the velocity magnitude scaled by $U$ : (a) $Q=1000 \mathrm{~s}^{-2}$; (b) $Q=5000 \mathrm{~s}^{-2}$.
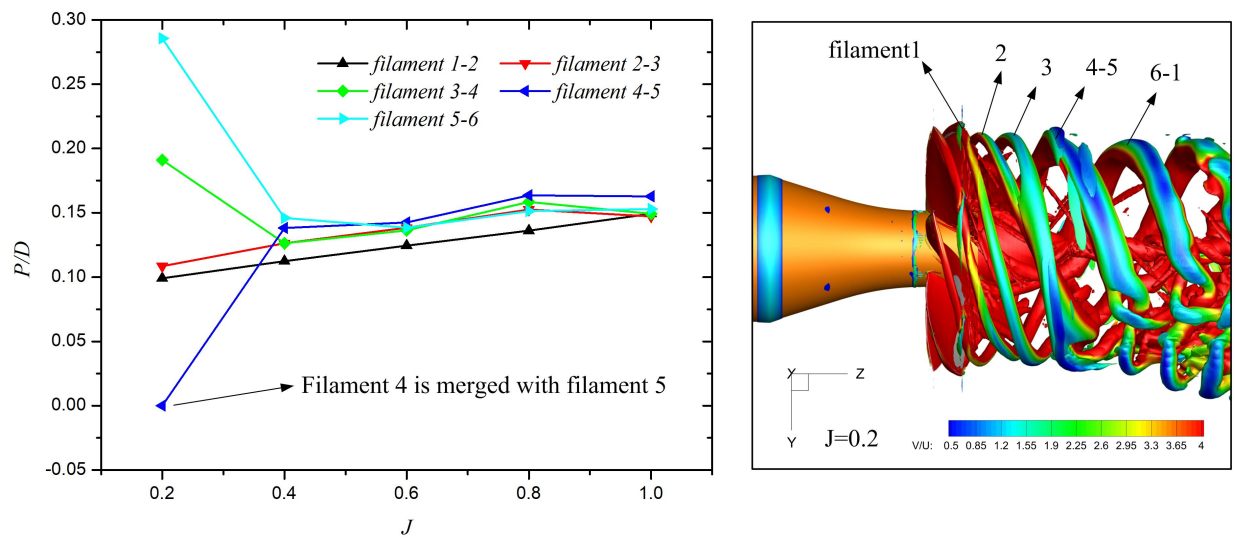

FIG. 18. Helical pitch ratio of tip filaments, $P / D$, for different values of $J$.

$t_{0}+\frac{1}{2} T$ for $J=0.2$. As can be seen in Fig. 21(a), three large tip vortices are formed, each of which is generated by the merger of two adjacent tip filaments (of filaments 1and 2, of filaments 3 and 4 , and of filaments 5 and 6 , respectively). The specific merger process can be seen in Fig. 20. The merger results in a clear increase in the helical pitch ratio of tip filaments, $P / D$ (see $J=0.2$ in Fig. 18). In addition, the next $P / D$ becomes zero owing to the merger. It should be mentioned here that the number of filaments in Fig. 18 is different from that in Figs. 20 and 21.

Here, the merger of vortices is similar to the "multistep" grouping mechanism observed in the experiment by Felli et $a l .{ }^{?}$ The difference is that the merged vortex can only remain stable for a very short distance, after which it splits into the 


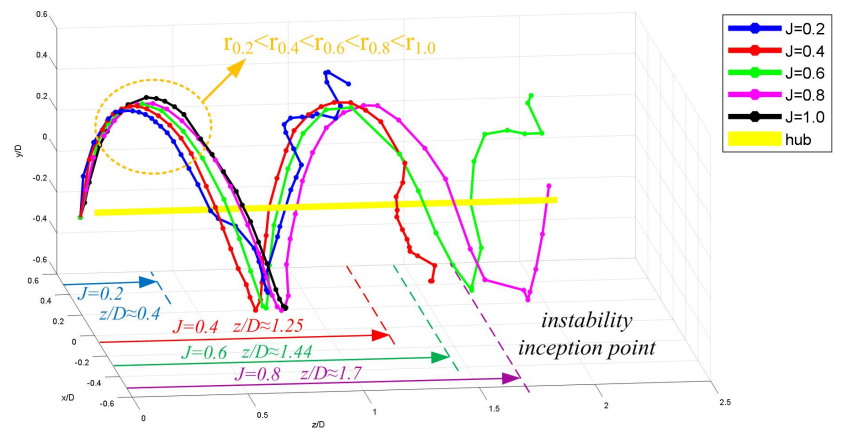

FIG. 19. Three-dimensional trajectory of a single filament.

two original tip filaments again owing to the interaction between tip vortices. After the splitting of the three merged vortices, a complex overlap interaction mode arises between the six filaments, and finally all of them gradually break down. For the six-bladed propeller in this study, there is no multistep grouping mechanism as captured by Felli et al., ? but a "merger-splitting" process does occur. We speculate that the reason for this is that the number of rotor blades $(n=6)$ is greater than that $(n=2,3$, or 4$)$ in the Felli et al. experiment, leading to a much smaller spiral-to-spiral distance than in the case of the E779a propeller. As shown in Fig. 18, $P / D$ for $J=0.2$ is much smaller than in the other cases, which causes a strong interaction between tip vortices. This merger-splitting phenomenon occurs only for $J=0.2$, not for $J=0.4$ or 0.6 , which confirms that the mutual-inductance instability and its underlying mechanism depend on the spiral-to-spiral distance, i.e., the helical pitch ratio of tip filaments.

From the above discussion, it can be seen that the roll-up process of the trailing edge vortex does not affect the stability of the tip vortex for $J=0.8$. Therefore, it might be asked what role does the roll-up process play in the case $J=0.2$ ?

The contours of the vorticity magnitude in different $z$ slices for $J=0.2$ are shown in Fig. 22. Compared with the case $J=$ 0.8 , fully developed roll-up occurs more rapidly, and the trailing edge is in contact with the next tip vortex at $z / D=0.15$ (for $J=0.8$, contact occurs at about $z / D=0.4$ ). Therefore, the smaller the value of $J$, the more rapid is the roll-up of the trailing edge vortex. At $z / D=0.3$, after the trailing edge vortex has come into contact with the next tip vortex, the angular displacement continues to increase, until the trailing edge vortex has contacted the second tip vortex and mutual-inductance instability of the tip vortices occurs. The six tip vortices are no longer uniformly distributed, but move closer to each other [Fig. 22(e)], merge with adjacent tip filaments [Fig. 22(f)], and each splits again into two isolated tip vortices [Fig. 22(g)], as discussed above.

Figure 23 shows the instantaneous flow field in $y-z$ plane of the $\mathrm{R}$ model for $J=0.2$. The low-pressure regions of the tip vortex are marked with red circles in Fig. 23(a), and it can be seen that the distance between adjacent low-pressure regions is clearly smaller than in the case $J=0.8$, which is consistent with the trend of change of $P / D$. Furthermore, the distribution of low-pressure regions exhibits an obvious asymmetry, which is caused by the merger and splitting of tip vortices. The contours of axial velocity [Fig. 23(b)] and vorticity magnitude [Fig. 23(c)] also exhibit the same asymmetry caused by mutual-inductance instability of the tip vortices.

The wake of the trailing edge vortex can be seen clearly at $z / D=0-0.5$. However, with the development of flow downstream, the trailing edge vortex gradually twists at $z / D=0.5-$ 1.5 and breaks down rapidly in the range $z / D>1.5$. The mutual inductance between tip vortex and trailing edge vortex further stimulates the instability of the tip vortex. Figure 24 shows the contours of axial velocity and vorticity magnitude for $J=0.4$ and 0.6. From a comparison of Fig. 23(c) with Figs. 24(c) and 24(d), we can see that the trailing edge vortex wake continues to gradually extend further downstream with increasing $J$.

The contours of the vorticity magnitude in the slice $z / D=$ 0.15 for different values of $J$ are shown in Fig. 25. For $J=0.2$, the wake of the trailing edge vortex is almost completely in contact with the next vortex. For $J=0.4$ and 0.6 , there is strong bending of the tip of the trailing wake owing to attraction by the next tip vortex. However, the bending of the trailing wake is clearly weaker for $J=0.8$ and 1.0. Hence, the smaller the value of $J$, the faster is the roll-up of the trailing edge vortex.

As discussed above, the pitch ratio $P / D$ increases gradually with increasing $J$. As a result, the multiple interactions between tip vortices become weak, which delays the full development of the roll-up process and finally reduces the likelihood of instability of the tip vortex.

As for the hub vortex, as shown in Fig. 23, for $J=0.2$, it always remains unstable after shedding from the hub. It should be noted that there is no trailing root vortex for $J=0.2$. For $J$ in the range $0.4-1.0$, the hub vortex undergoes the same form of evolution, maintaining spiral twisted form in the near field and then gradually becoming stable, as shown by its straight line motion. This behavior is completely different from that of the hub vortex of the E779A propeller?' As for the R model, as discussed earlier, the spiral twisted form of the hub vortex in the near-field region is caused by the presence of the trailing root vortex. As shown in Fig. 17, the breakdown point of the trailing root vortex moves further downstream with increasing $J$. For $J=1.0$, even though the tip vortex break down quickly, the trailing root vortex survive for the longest distance compared with the other cases. More importantly, the twist region of the hub vortex maintains the same distance from the trailing root vortex. For $J=0.6$ and 0.8 , breakdown of the trailing root vortex also occurs almost simultaneously with the disappearance of the twist in the hub vortex. Hence, the presence of trailing root vortices is the reason why the hub vortex can continue to twist without instability arising. The period of the hub vortex oscillation will be discussed below.

\section{Vortex dynamics}

To study the dynamical behavior of the hub and tip vortices for the R model, an analysis of the turbulence kinetic energy (TKE) is carried out. Probes are located at different stream- 


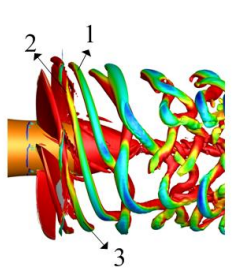

(a) $\mathrm{t}_{0}$

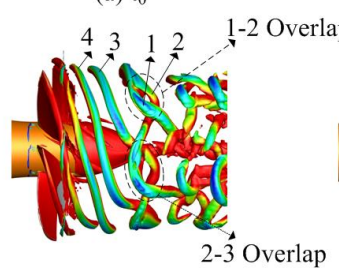

(e) $t_{0}+1 / 2 T$

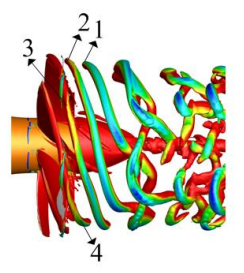

(b) $\mathrm{t}_{0}+1 / 8 \mathrm{~T}$

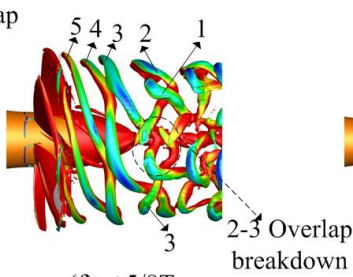

(f) $\mathrm{t}_{0}+5 / 8 \mathrm{~T}$

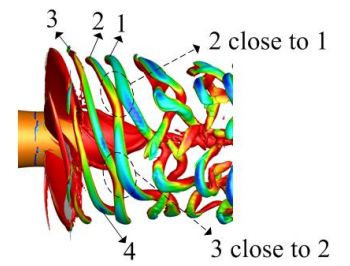

(c) $\mathrm{t}_{0}+1 / 4 \mathrm{~T}$

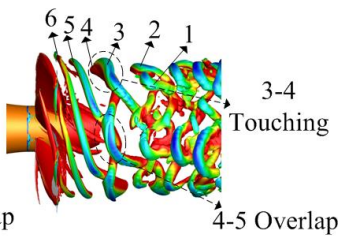

$(\mathrm{g}) \mathrm{t}_{0}+3 / 4 \mathrm{~T}$

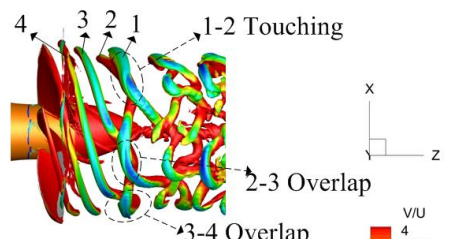

(d) $\mathrm{t}_{0}+3 / 8 \mathrm{~T}$ and merge

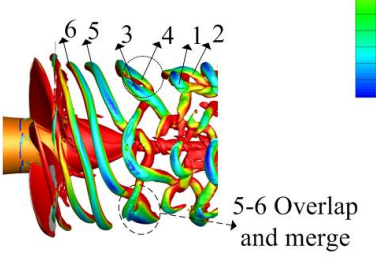

(h) $\mathrm{t}_{0}+7 / 8 \mathrm{~T}$

FIG. 20. Time evolution of the tip vortex overlap-forward phenomenon for $J=0.2\left(Q=5000 \mathrm{~s}^{-2}\right)$.

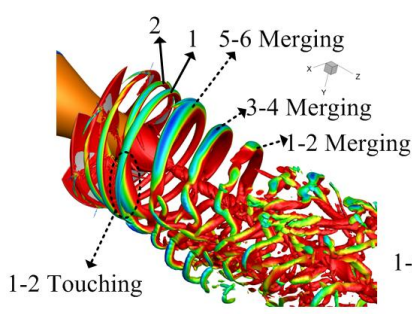

(a) $\theta=0^{\circ}$

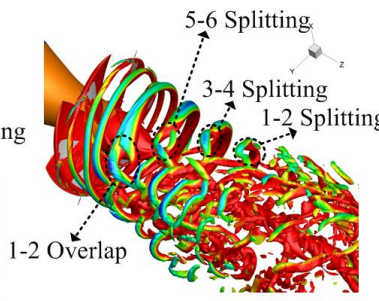

(b) $\theta=-60^{\circ}$

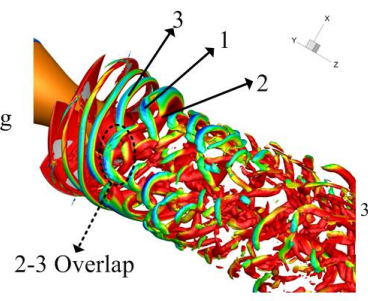

(c) $\theta=-120^{\circ}$

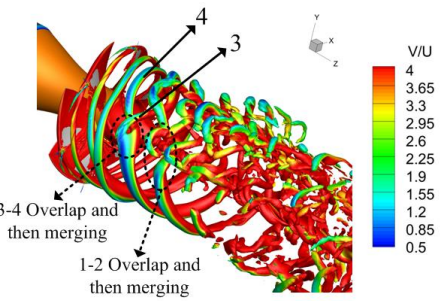

(d) $\theta=-180^{\circ}$

FIG. 21. Spatial evolution of the tip vortex overlap-forward phenomenon at $t=t_{0}+\frac{1}{2} T$ for $J=0.2\left(Q=5000 \mathrm{~s}^{-2}\right)$.

wise positions behind the tip of the rotor (probes $\mathrm{P} 1, \ldots, \mathrm{P} 8$ at $r / D=0.45$ ) and hub (probes $\mathrm{P} 9, \ldots, \mathrm{P} 16)$, as shown in Fig. 26. The time histories acquired at these probes are shown in Figs. 27 and 28.

The different unstable transition forms of the tip vortex for different values of $J$ can clearly be seen in Fig. 27. For $J=0.2$, the TKE values are chaotic at $\mathrm{P} 1, \ldots, \mathrm{P} 8$, which indicates that the tip vortex always remains unstable at $z / D=0.5$. This is consistent with the evolution of tip vortex discussed earlier (see Fig. 17). For $J=0.6$, the TKE clearly exhibits periodic characteristic at P1. From about P3 $(z / D=1.5)$ onward, the TKE signal begins to lose its purely periodic characteristic and exhibits nonperiodicity and randomicity. This is consistent with the location of the inception point of tip vortex instability for $J=0.6$ (at about $z / D=1.44$ ). For $J=1.0$, which is similar to the case $J=0.8$, the TKE signals at P1, ., P8 always exhibit periodicity in time.

For the region of the hub vortex, the time histories of TKE at P9, .., P16 (behind the hub) are shown in Fig. 28. For $J=$ 0.2 , the TKE signals at P9, ., P16 are always chaotic. Hence, the hub vortex for $J=0.2$ always remains unstable. For $J=$ 0.6 , the TKE signals at P14, ., P16 remain nearly constant in time, which corresponds to the stable region of the hub vortex following the gradual disappearance of distortion after $z / D>$ 3 . For $J=1.0$, the unstable region survives longer than for $J=0.8$. The hub vortex retains a double-helical progressive motion until P16.

A comparison of TKE time histories for different values of $J$ at $\mathrm{P} 1$ and $\mathrm{P} 9$ is shown in Fig. 29. The variations for $J=0.6$ and 1.0 have the same period, $\frac{1}{6} T$ (where $T$ is the rotation period of the hub: $\frac{1}{20} \mathrm{~s}=0.05 \mathrm{~s}$ ), whereas for $J=0.2$, a distinct period $\frac{1}{3} T$ is observed. As discussed before, this is a consequence of the merger of each pair of adjacent tip filaments. Hence, the period of tip vortex shedding for the $\mathrm{R}$ model is $(1 / n) T$ (where $n$ is the number of rotor blades) in most cases except in some cases of low $J(J \leq 0.2)$. At P9, the TKE value for $J=0.2$ is more like a random signal, whereas that for $J=1.0$ has an approximately straight line form, which is consistent with the earlier discussion of hub vortex stability.

A frequency analysis of TKE for different values of $J$ is carried out, as shown in Figs. 30 and 32. The power spectral density (PSD) of the TKE at probes P1, ., P16 is computed. First, for the near field of the tip vortices $(\mathrm{P} 1, \ldots, \mathrm{P} 3)$, for all three values of $J$, the PSD has obvious peaks at $f_{\mathrm{BPF}}$ (the blade passing frequency) and its harmonics. In addition, as can be seen in Fig. 29, the TKE at P1 for $J=0.2$ has a peak at $0.5 f_{\mathrm{BPF}}$ and its harmonics $\left(f_{\mathrm{BPF}}\right.$ and $\left.1.5 f_{\mathrm{BPF}}\right)$, which corresponding to the $\frac{1}{3} T$ period resulting from the merger of tip vortices. In the far-field region ( $\mathrm{P} 5, \ldots, \mathrm{P} 7)$, the peaks at $n f_{\mathrm{BPF}}$ gradually disappear and are replaced with several peaks at $f_{N}, 2 f_{N}$, and $4 f_{N}$. Furthermore, at P7 and P8, the spectral lines show nearbroadband characteristics. 


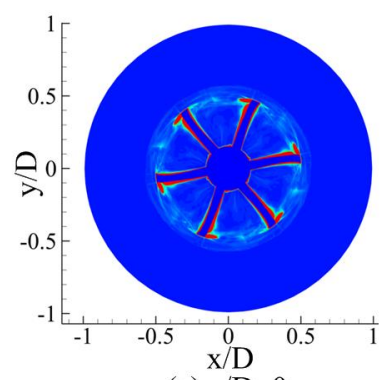

(a) $\mathrm{z} / \mathrm{D}=0$

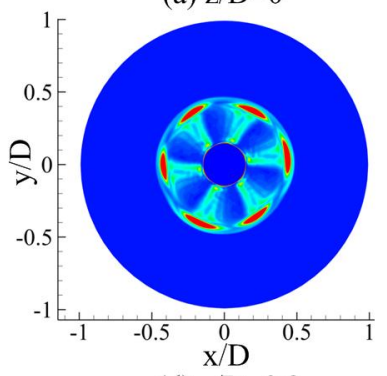

(d) $\mathrm{z} / \mathrm{D}=0.2$

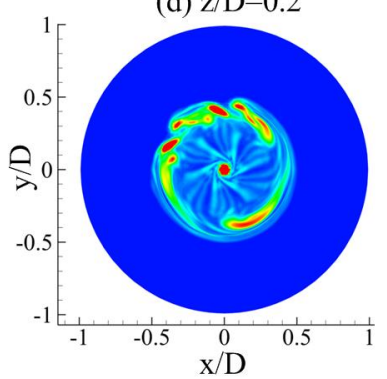

(g) $\mathrm{z} / \mathrm{D}=0.5$

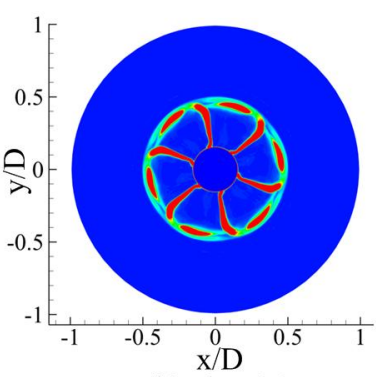

(b) $\mathrm{z} / \mathrm{D}=0.1$

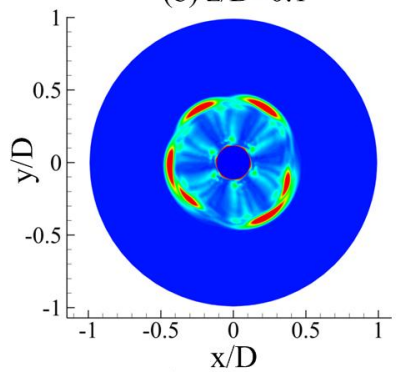

(e) $\mathrm{z} / \mathrm{D}=0.3$

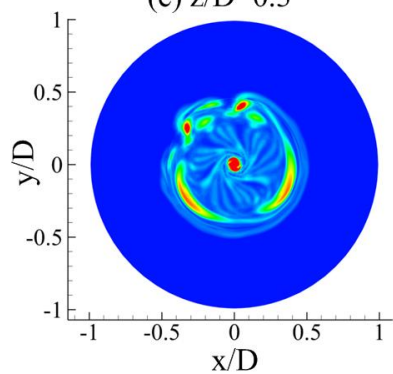

(h) $\mathrm{z} / \mathrm{D}=0.6$

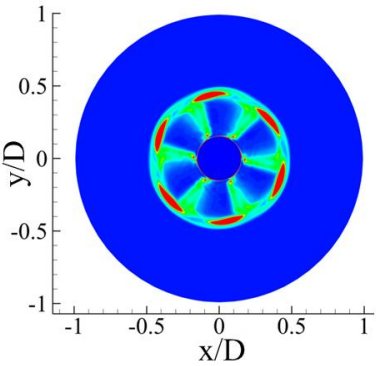

(c) $\mathrm{z} / \mathrm{D}=0.15$

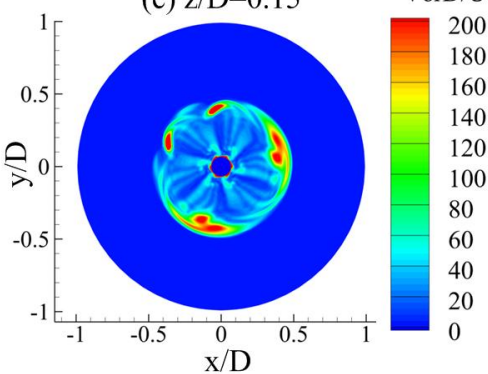

(f) $\mathrm{z} / \mathrm{D}=0.4$

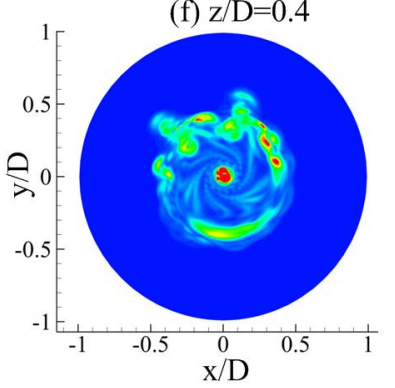

(i) $\mathrm{z} / \mathrm{D}=1$

FIG. 22. Contours of the vorticity magnitude for $J=0.2$ in different $z$ slices, normalized with $U / D$

This energy transfer process from blade harmonics to shaft harmonics has been verified experimentally by Felli et al.. To show the energy transfer process more clearly, the amplitudes of the PSD at $f_{N}$ to $6 f_{N}\left(=f_{\mathrm{BPF}}\right)$ are shown in Fig. 31 . As can be seen, the streamwise evolution of the PSD of the TKE demonstrates a mechanism of energy relocation from blade harmonics to shaft harmonics $n f_{\mathrm{BPF}}$. More specifically, the amplitude of the PSD at the fundamental frequency $f_{\mathrm{BPF}}$ decreases gradually with increasing shaft frequency $f_{N}$ and with increasing $z / D$. In addition, some differences are found. For a four-bladed propeller, the energy transfer is divided into two steps: (i) the first energy transfer from $f_{\mathrm{BPF}}$ to $0.5 f_{\mathrm{BPF}}$; (ii) the second energy transfer from $0.5 f_{\mathrm{BPF}}$ to $f_{N}$. Corresponding to this two-step process is a group of multiple tip vortices, as described by Felli et al..?

For the R model studied here, such a two-step energy transfer process is not found. The PSD at $f_{N}$ to $5 f_{N}$ exhibits a different trend of change with $J$. For all values of $J$, the contribution at the fundamental frequency $f_{\mathrm{BPF}}$ gradually disappears. The greater the value of $J$, the steeper is the slope of decrease of PSD at $f_{\mathrm{BPF}}$.

For $J=0.6$, at $z / D=1.0-2.5$, all the contributions at $f_{N}$ to $5 f_{N}$ increase gradually. Then, at $z / D=2.5-4$, the PSD at
$f_{N}$ continues to increase, while the components at $2 f_{N}$ to $5 f_{N}$ decrease rapidly. However, for $J=1.0$, the trend of energy change is quite different. In the near-field region $z / D=0.5-$ 2 , all the contributions at $f_{N}$ to $5 f_{N}$ clearly decrease, which is consistent with the rapid breakdown of the tip vortex observed in Fig. 17. In the far-field region $z / D=2-4$, only the contribution at $f_{N}$ is increased. For $\boldsymbol{J}=0.2$, the PSD changes chaotically. In the range $z / D=0.5-4$, the PSD amplitude at $f_{N}$ to $4 f_{N}$ experiences periodic fluctuations. We speculate that these may be caused by the merger and separation of tip vortices. This also confirms the conclusion by Felli et al. ${ }^{?}$ that the energy transfer process is closely related to the tip vortex evolution.

In general, an energy transfer of tip vortices from blade harmonics to shaft harmonics is found. The specific mechanism of this energy transfer depends on $J$ and is related to tip vortex evolution.

For the hub vortex, the PSD spectra at P9, ., P16 are shown in Fig. 32. For $J=0.2$, the PSD spectrum is basically a broadband spectrum corresponding to instability of the hub vortex. For $J=0.6$ and 1.0 , several peaks at $f_{N}$ to $4 f_{N}$ can be seen in Figs. 32(b) and 32(c). Therefore, the characteristic frequency of evolution for the hub vortex should be $f_{N}$ and 


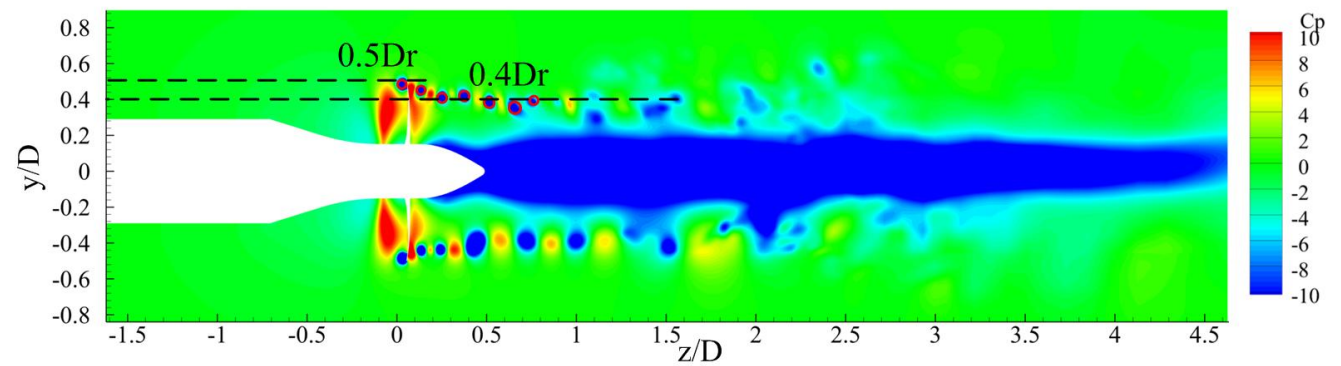

(a)

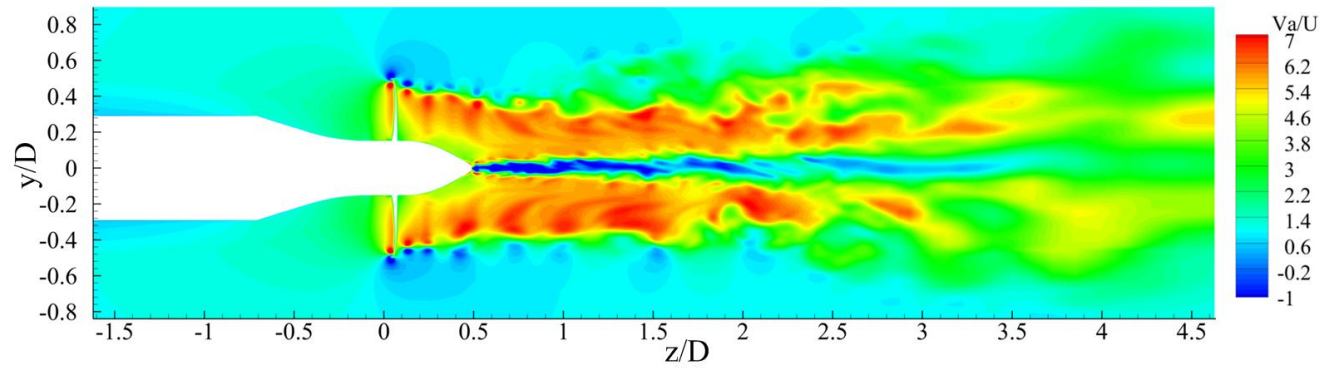

(b)

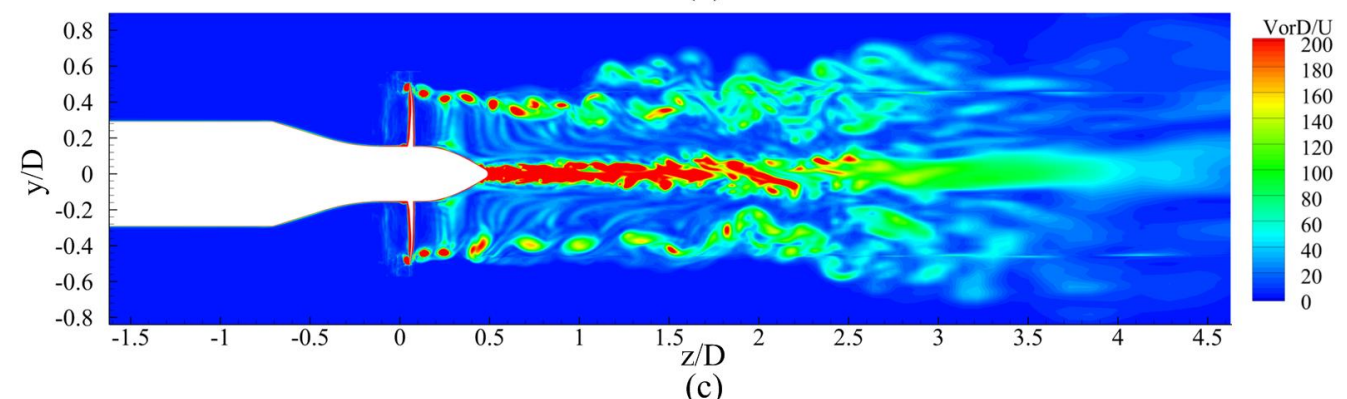

FIG. 23. Instantaneous flow field in the $y-z$ plane of the R model for $J=0.2$ : (a) pressure coefficient; (b) axial velocity; (c) vorticity magnitude. The axial velocity is scaled by $U$, and the vorticity magnitude is normalized with $U / D$.

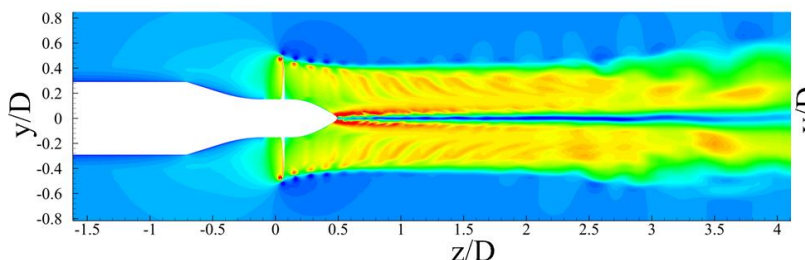

(a)

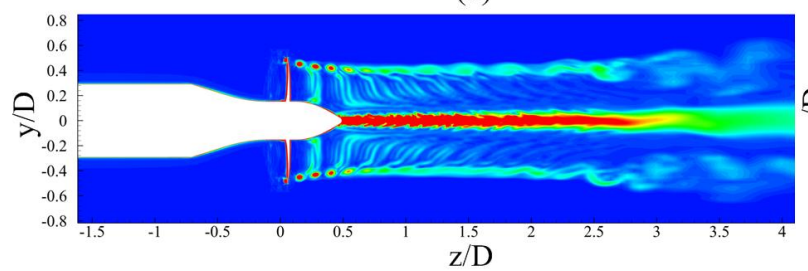

(c)

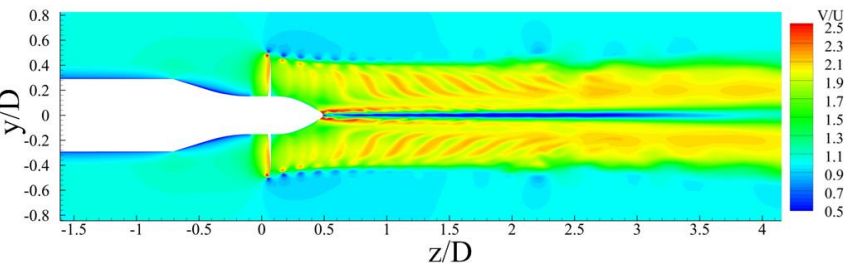

(b)

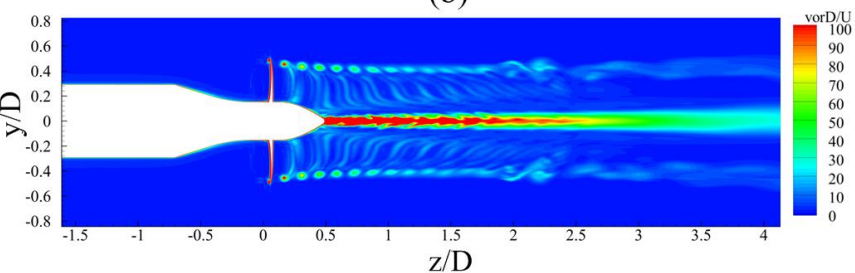

(d)

FIG. 24. Instantaneous flow field in $y-z$ plane of the R model for $J=0.4$ (left) and $J=0.6$ (right): (a) axial velocity; (b) vorticity magnitude. The axial velocity is scaled by $U$, and the vorticity magnitude is normalized with $U / D$.

the period of double-helical motion of the hub vortex should equal one propeller revolution $T$ (the hub rotation period).

\section{CONCLUSIONS}

In this paper, DES studies of vortex instabilities in the wake of a pre-swirl pumpjet propulsor (PJP) have been carried out. 


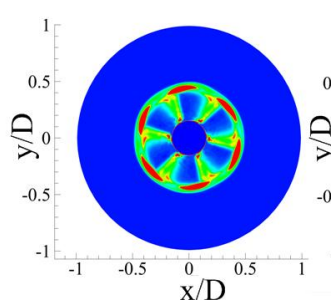

(a) $\mathrm{J}=0.2$

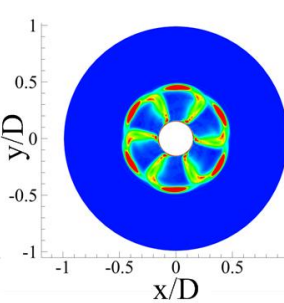

(b) $\mathrm{J}=0.4$

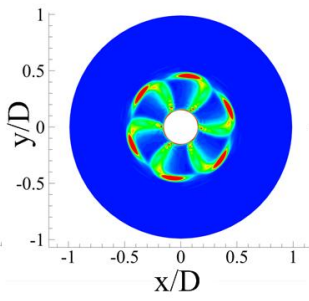

(c) $\mathrm{J}=0.6$

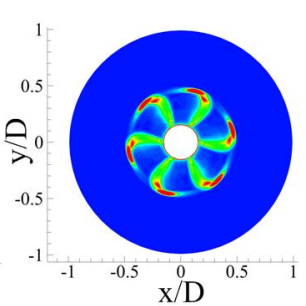

(d) $\mathrm{J}=0.8$

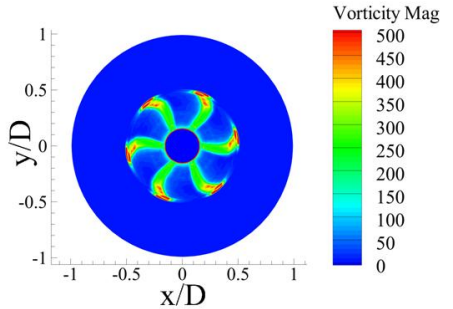

(e) $\mathrm{J}=1.0$

FIG. 25. Contours of vorticity magnitude in the $x-y$ plane at $z / D=0.15$, normalized using $U / D$.

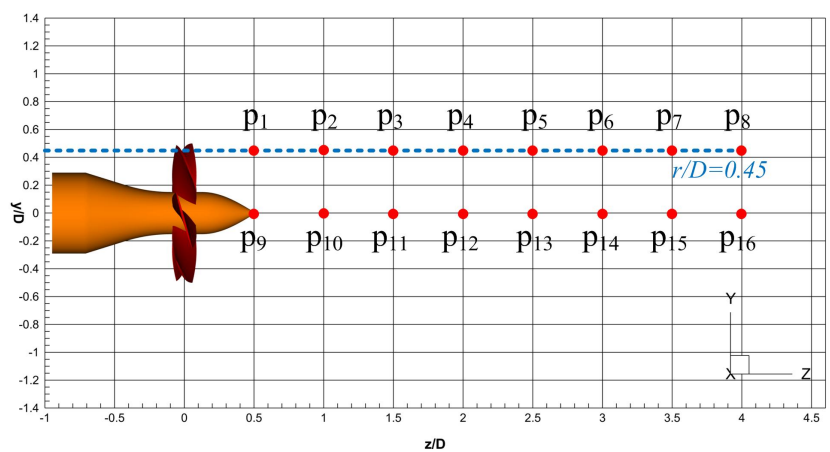

FIG. 26. Probe locations for the dynamical analysis of the tip and hub vortices for the $\mathrm{R}$ model.

To separate the roles played by the rotor, stator, and duct in determining the vortex structure of a PJP, three models have been established: the R model (rotor only), the R-D model (rotor within a duct), and the PJP model (rotor and stator within a duct). In this paper, only the three-dimensional vortex structure of a single rotor has been considered: the R-D and PJP models will be discussed in detail in Part 2.

The instantaneous wake vortex structure, the evolution in time and space of the tip and hub vortices, the transition from stability to instability of tip and hub vortices, the instability trigger mechanism, the unique roll-up process of the R model, and the vortex dynamics have been analyzed systematically.

The numerical results reveal the following:

1. A unique trailing tip vortex is found in the wake of the $\mathrm{R}$ model, together with the tip vortex and trailing root vortex. This vortex, appearing as spiral filaments within the tip filaments, is caused by premature shedding of tip vortices in the $\mathrm{R}$ model compared with a normal single propeller.

2. The roll-up process of the trailing edge vortex of the $\mathrm{R}$ model with broad-tipped rotor blades is slightly different from the process for a normal single propeller. There is mutual attraction between the trailing root vortex and the trailing tip vortex. The trailing tip vortex takes over the role of the tip vortices, which eliminates the effects of mutual interaction of two adjacent tip vortices and eventually leads to stabilization of the tip vortices for high values of $J$.

3. A unique multi-inductance instability mode of the tip vortex for low $J$, called the overlap-forward phenomenon, is proposed. This phenomenon is quite different from the normal leapfrogging phenomenon that occurs with a single propeller.

4. It is found that the instability of the tip vortex depends not only on the spiral-to-spiral distance but also on the the highest-efficiency point of the propeller. Specifically, the spiral-to-spiral distance increases gradually with increasing $J$. The instability inception point of the tip vortex moves farther downstream with increasing $J$, and, when $J$ is greater than the highest-efficiency point of the propeller $(J>0.8$ here $)$, the stable length of the tip vortices drops sharply.

5. A quite different instability mode of the hub vortex is found for the $\mathrm{R}$ model. Under most working conditions $(0.4<J<1.0)$, the hub vortex remains coherent for the entire length of the simulation. In addition, the hub vortex takes the helix twisted form at near field behind the hub. After that, the twist disappears gradually and the hub vortex keeps stable until breakdown in the far field. The usual double-helix breakdown and instability of the hub vortex do not occur. The existence of trailing root vortices is the reason why the hub vortex can maintain its twist without the occurrence of instability. The region where the hub vortex maintains its twist is basically the same as the region where trailing root vortices exist. The twist region survives for a longer distance with increasing $J$.

6. The fundamental frequencies of TKE fluctuations in the tip vortex region are the rotor blade passing frequency and its harmonics, while the fundamental frequency of the hub vortex is the shaft rotation frequency. The energy transfer process of tip vortices from blade harmonics to shaft harmonics depends on $J$ and is related to the spatial evolution of the tip vortices.

\section{ACKNOWLEDGMENTS}

This work is supported by the National Natural Science Foundation of China under Project Nos. 51979226, 

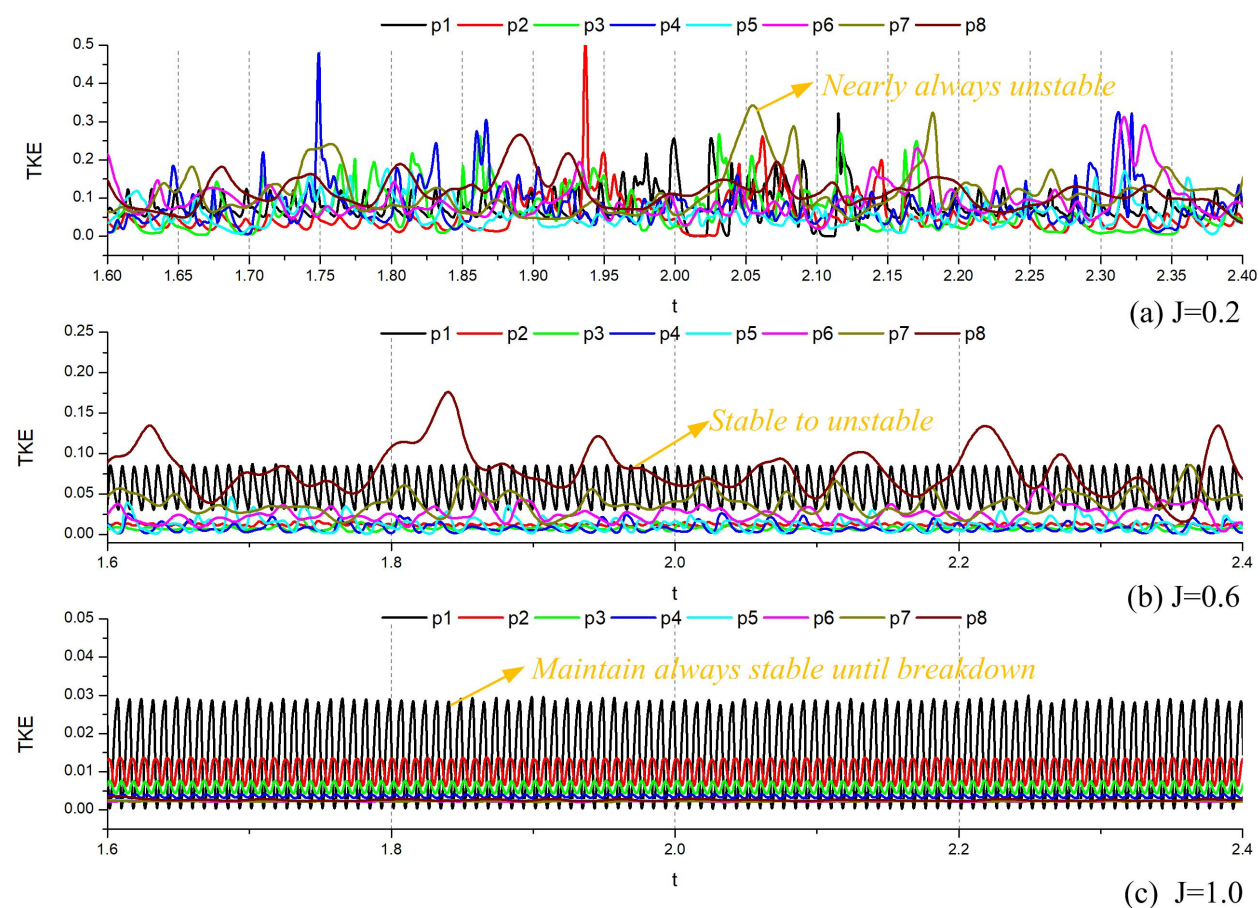

FIG. 27. Time histories of TKE at P1, ., P8 for the R model: (a) $J=0.2$; (b) $J=0.6$; (c) $J=1.0$.
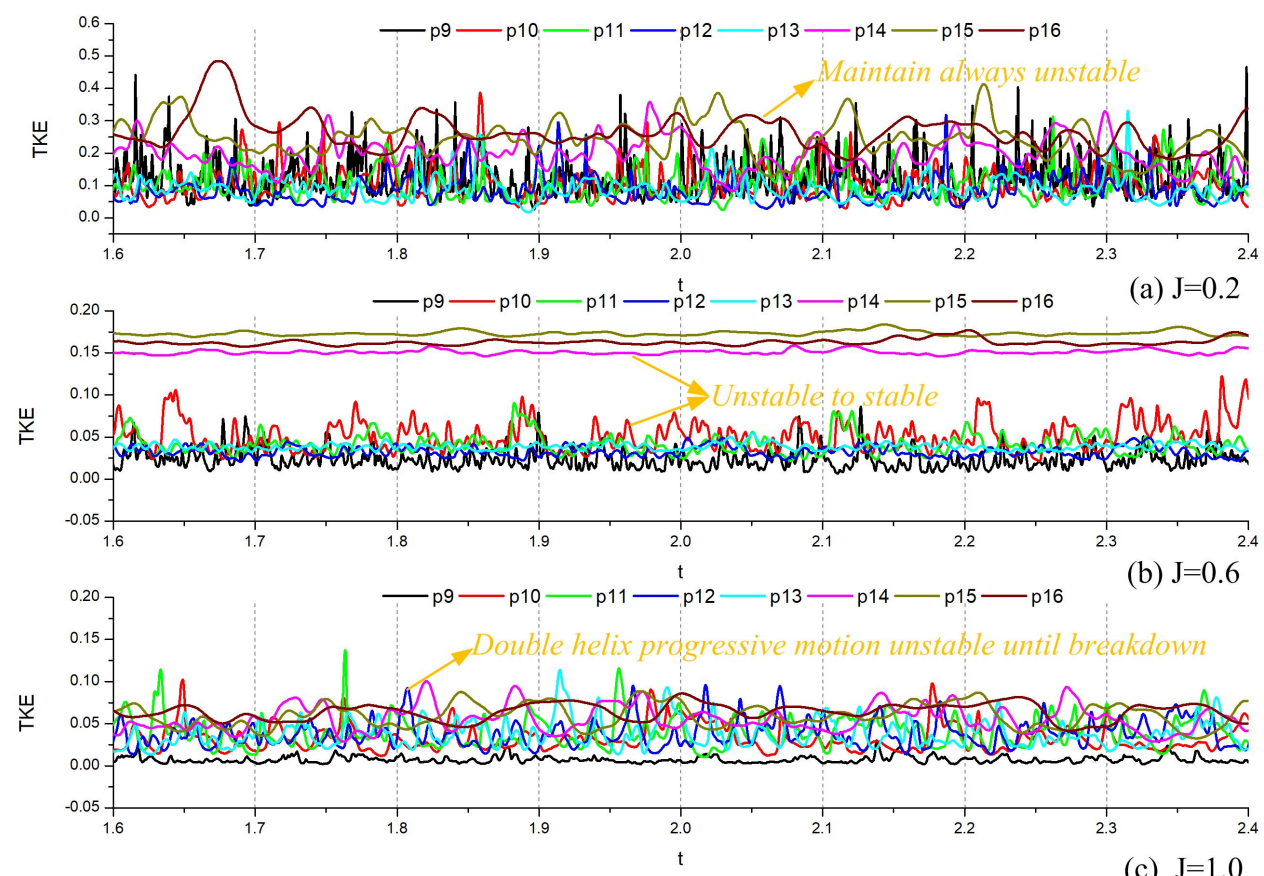

FIG. 28. Time histories of TKE at P9, .., P16 for the R model: (a) $J=0.2$; (b) $J=0.6$; (c) $J=1.0$.

51879220 , and 51709229 and by the Fundamental Research Funds for the Central Universities under Project Nos. 3102019 HHZY030019 and 3102020HHZY030018.

\section{DATA AVAILABILITY}

The data that support the findings of this study are available within the article. Additional data that support these findings 

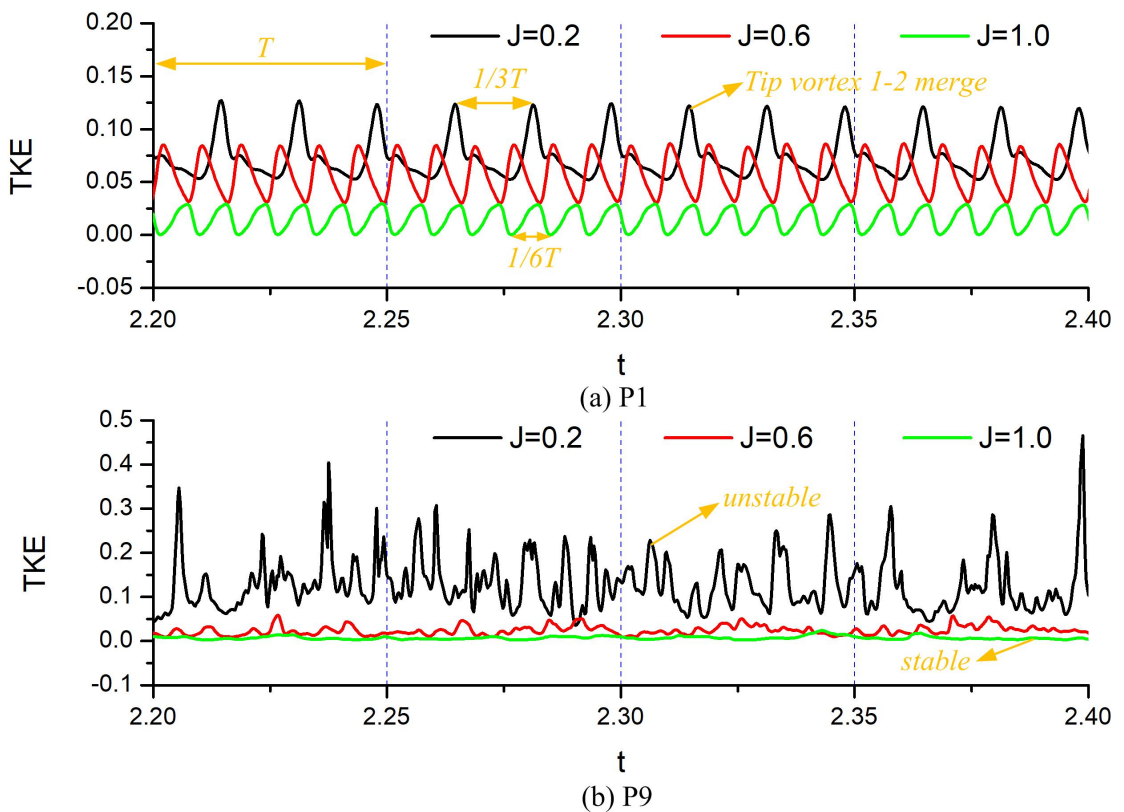

FIG. 29. Comparisons of time histories for different values of $J$ : (a) at P1; (b) at P9.

are available from the corresponding author upon reasonable request.

\section{REFERENCES}



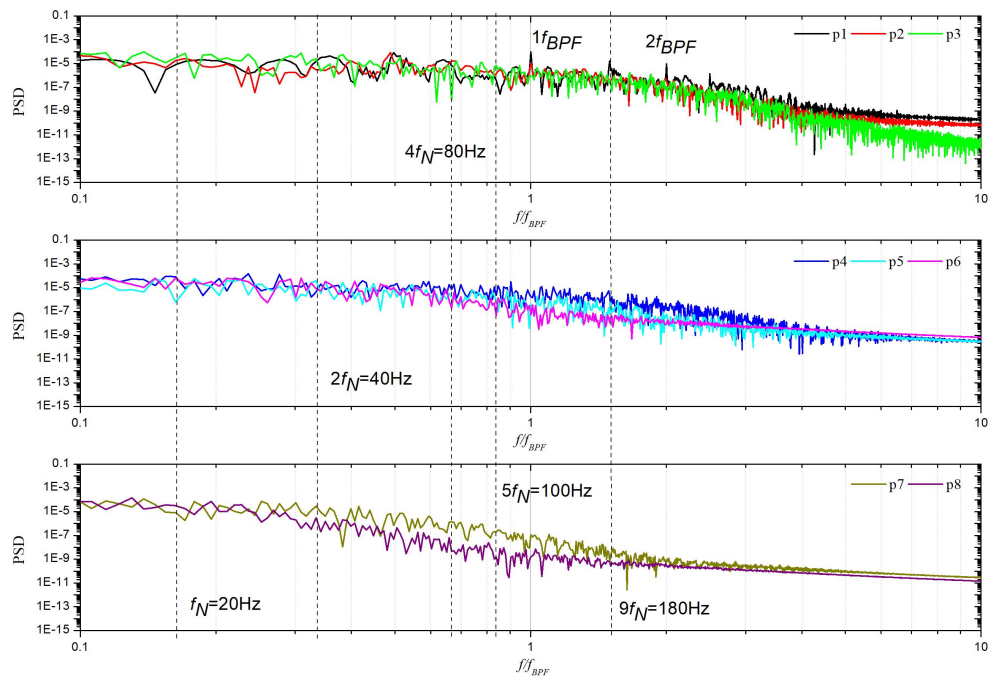

(a) $\mathrm{J}=0.2$
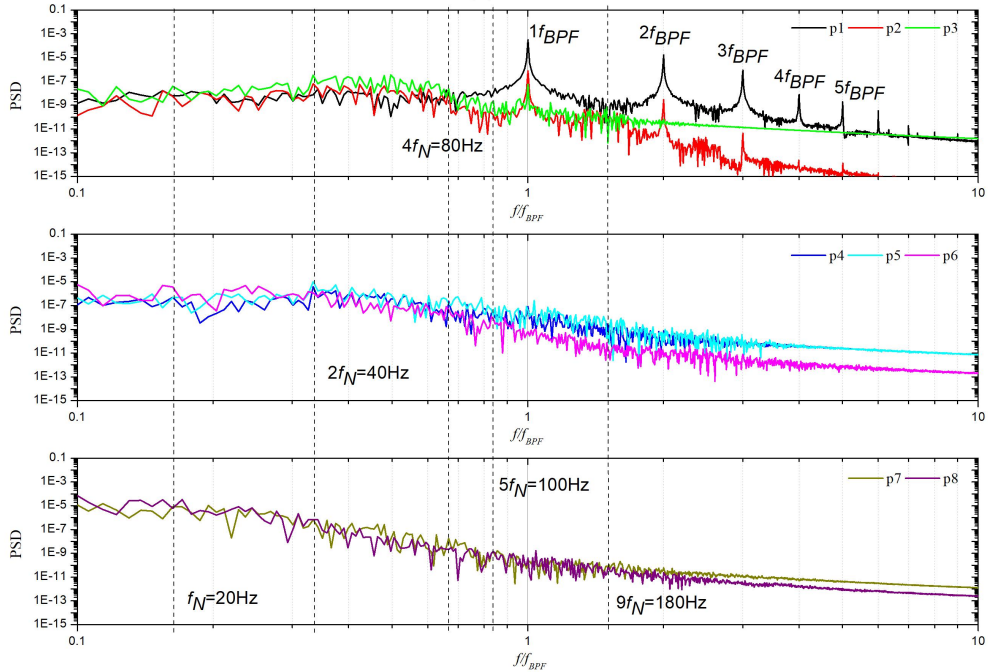

(b) $\mathrm{J}=0.6$

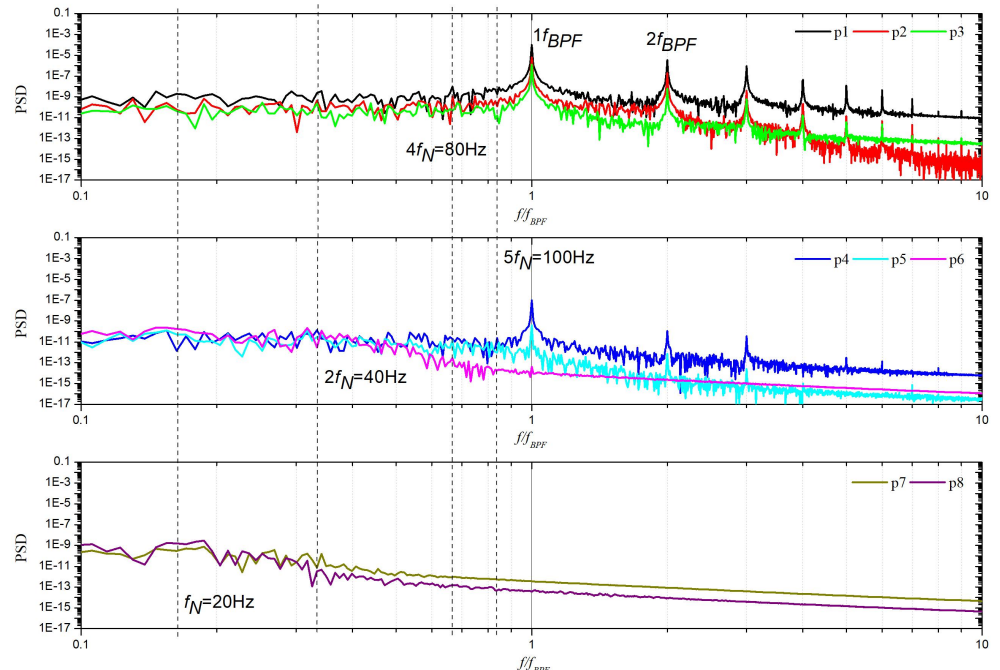

(c) $\mathrm{J}=1.0$

FIG. 30. Power spectra of the turbulence kinetic energy for the R model at P1, ., P8: (a) $J=0.2$; (b) $J=0.6$; (c) $J=1.0$. 


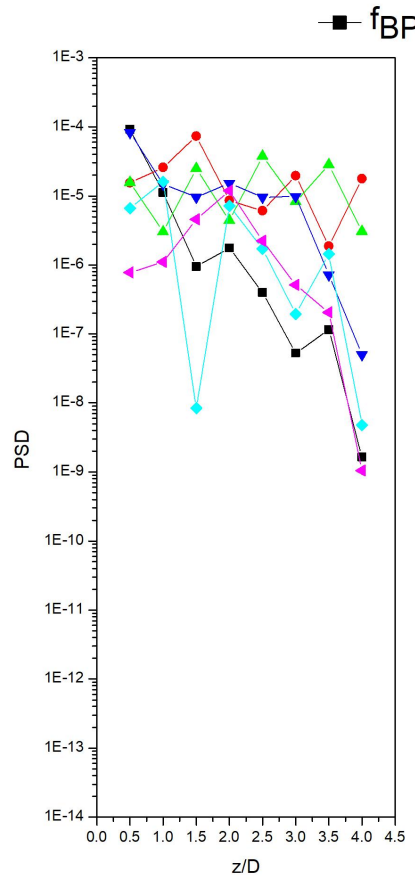

(a) $\mathrm{J}=0.2$
$-f_{B P F}-f_{N}-2 f_{N}-3 f_{N}$

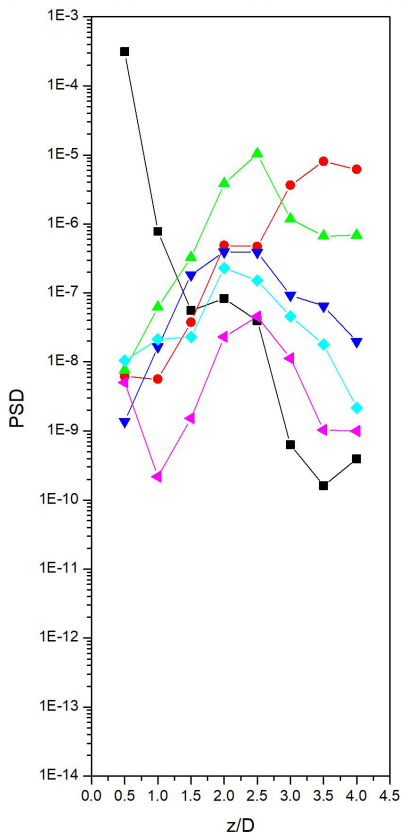

(b) $\mathrm{J}=0.6$

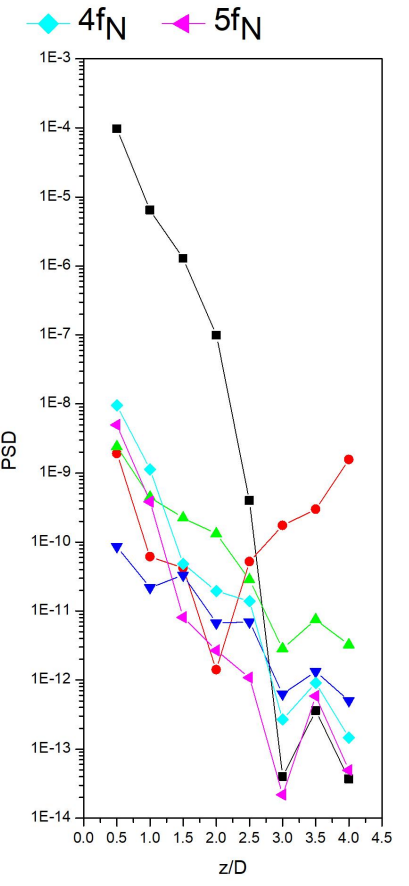

(c) $\mathrm{J}=1.0$

FIG. 31. Process of energy transfer at the tip vortices of the R model: (a) $J=0.2$; (b) $J=0.6$; (c) $J=1.0$. 

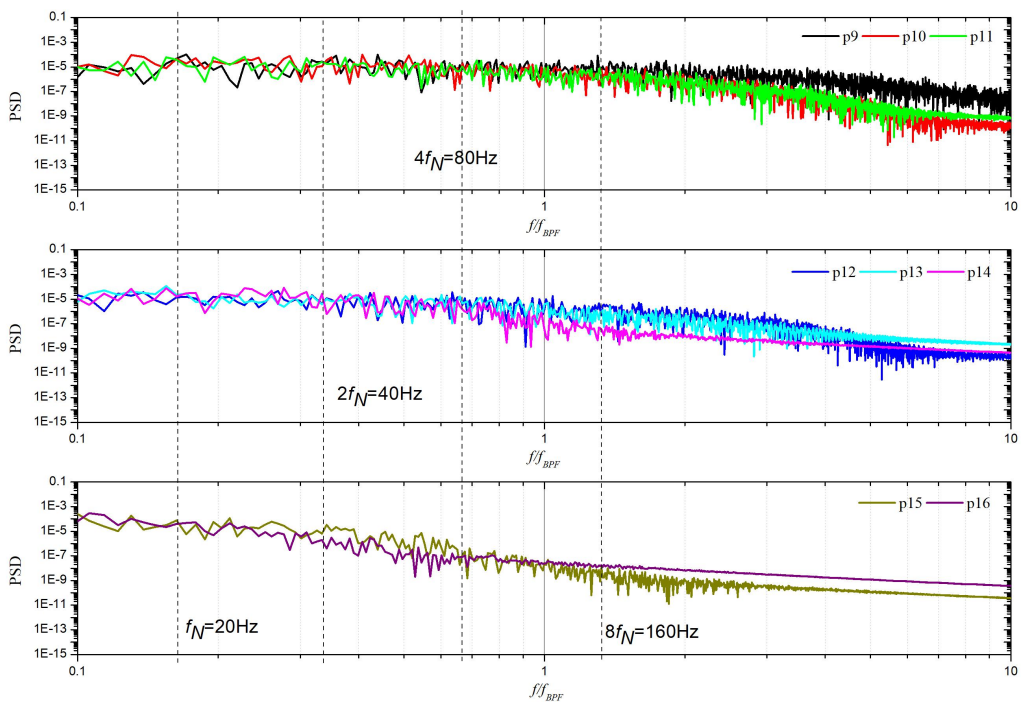

(a) $\mathrm{J}=0.2$
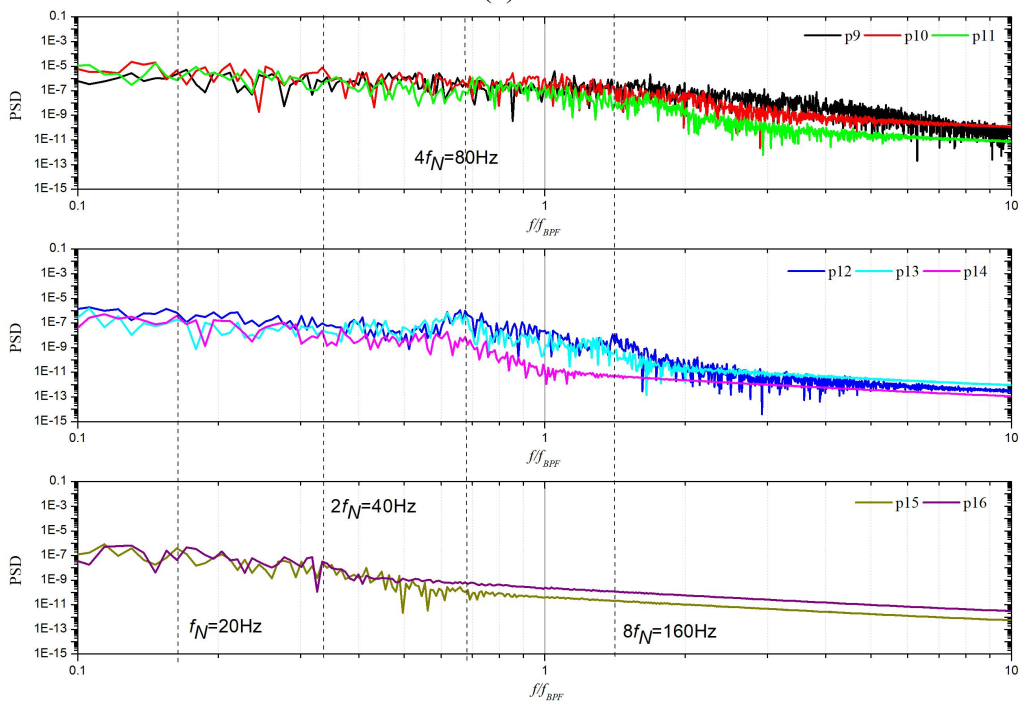

(b) $\mathrm{J}=0.6$
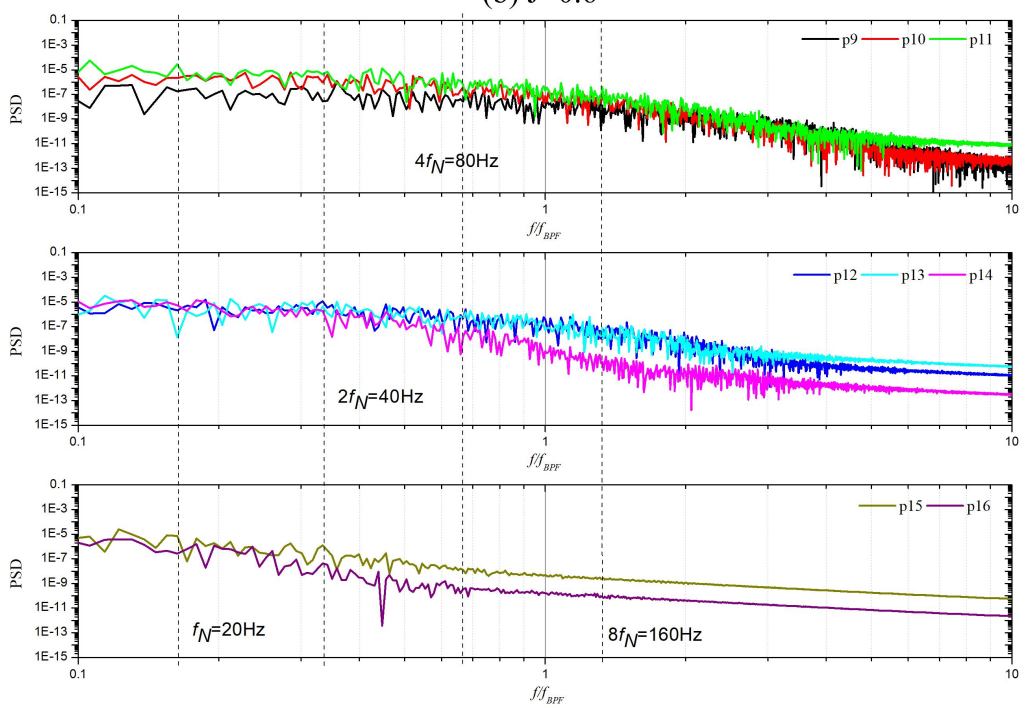

(c) $\mathrm{J}=1.0$

FIG. 32. Power spectra of the turbulence kinetic energy for the R model at P9, ., P16: (a) $J=0.2$; (b) $J=0.6$; (c) $J=1.0$. 